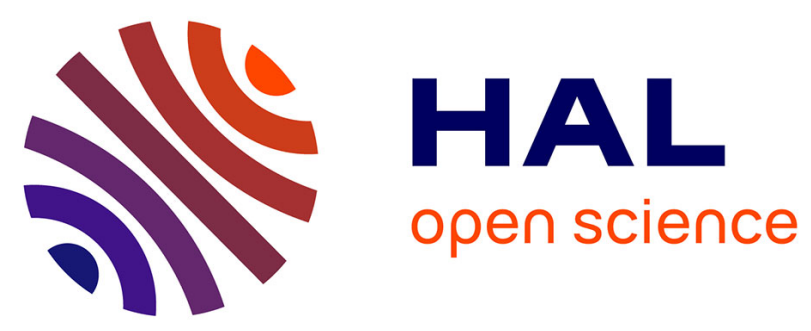

\title{
Development of fracture facets from a crack loaded in mode I+III: Solution and application of a model 2D problem
}

Jean-Baptiste Leblond, Joël Frelat

\section{> To cite this version:}

Jean-Baptiste Leblond, Joël Frelat. Development of fracture facets from a crack loaded in mode I+III: Solution and application of a model 2D problem. Journal of the Mechanics and Physics of Solids, 2014, 64, pp.133 - 153. 10.1016/j.jmps.2013.11.001 . hal-01436391

\section{HAL Id: hal-01436391 https://hal.sorbonne-universite.fr/hal-01436391}

Submitted on 16 Jan 2017

HAL is a multi-disciplinary open access archive for the deposit and dissemination of scientific research documents, whether they are published or not. The documents may come from teaching and research institutions in France or abroad, or from public or private research centers.
L'archive ouverte pluridisciplinaire HAL, est destinée au dépôt et à la diffusion de documents scientifiques de niveau recherche, publiés ou non, émanant des établissements d'enseignement et de recherche français ou étrangers, des laboratoires publics ou privés. 


\title{
Development of fracture facets from a crack loaded in mode I+III: solution and application of a model 2D problem
}

\author{
Jean-Baptiste Leblond *, Joël Frelat \\ UPMC Univ Paris 06, UMR 7190, Institut Jean Le Rond d'Alembert, Tour 65-55, 4 place \\ Jussieu, 75005 Paris, France
}

\begin{abstract}
It is experimentally well-known that a crack loaded in mode I+III propagates through formation of discrete fracture facets inclined at a certain tilt angle on the original crack plane, depending on the ratio of the mode III to mode I initial stress intensity factors. Pollard et al. (1982) have proposed to calculate this angle by considering the tractions on all possible future infinitesimal facets and assuming shear tractions to be zero on that which will actually develop. In this paper we consider the opposite case of well-developed facets; the stress field near the lateral fronts of such facets becomes independent of the initial crack and essentially 2D in a plane perpendicular to the main direction of crack propagation.

To determine this stress field, we solve the model $2 \mathrm{D}$ problem of an infinite plate containing an infinite periodic array of cracks inclined at some angle on a straight line, and loaded through uniform stresses at infinity. This is done first analytically, for small values of this angle, by combining Muskhelishvili (1953)'s formalism and a first-order perturbation procedure. The formulae found for the 2D stress intensity factors are then extended in an approximate way to larger angles by using another reference solution, and finally assessed through comparison with some finite element results.

To finally illustrate the possible future application of these formulae to the prediction of the stationary tilt angle, we introduce the tentative assumption that the 2D mode II stress intensity factor is zero on the lateral fronts of the facets. An approximate formula providing the tilt angle as a function of the ratio of the mode III to mode I stress intensity factors of the initial crack is deduced from there. This formula, which slightly depends on the type of loading imposed, predicts somewhat smaller angles than that of Pollard et al. (1982).
\end{abstract}

Keywords : Mode I+III; fracture facets; model 2D problem; analytical solution; finite element solution; prediction of tilt angle

$\bar{*}$ Corresponding author. 


\section{Introduction}

It is well-known that a crack, when loaded in mixed mode I+III, tends to propagate out of its original plane. This topic has been the subject of many experimental, numerical and theoretical papers.

The majority of these papers were of experimental nature; see the works of (Sommer, 1969; Knauss, 1970; Palaniswamy and Knauss, 1975; Hourlier and Pineau, 1979; Pollard et al., 1982; Suresh and Tschegg, 1987; Pollard and Aydin, 1988; Yates and Miller, 1989; Hull, 1993; Yates and Mohammed, 1994; Hubbard, 1995; Hull, 1995; Cooke and Pollard, 1996; Lazarus, 1997; Lazarus et al., 2001a,b, 2008; Lin et al., 2010; Goldstein and Osipenko, 2012), among others. All these experimental investigations have shown that the crack propagates through formation of small fracture "facets" (or "lances" in Sommer (1969)'s terminology) which may either abruptly "tilt" or gradually "twist" about the direction of propagation; the wording "crack front segmentation" is often used to designate the phenomenon. Two types of facets are in fact formed: in Hourlier and Pineau (1979)'s terminology, "type A" facets rotating in such a way that the local stress intensity factor (SIF) of mode I increases with the distance of propagation while that of mode III decreases; and "type B" facets rotating oppositely so that the local SIF of mode I decreases while that of mode III increases. It has been observed by Hourlier and Pineau (1979), followed by many other authors, that the crack propagates more along type A facets than along type B ones; in many cases type B facets are even totally absent. This observation suggests that the crack would ideally like to develop exclusively along type A facets, thus minimizing the presence of mode III along the front; and that type B facets, when present, are so only because they are "geometrically necessary" to join type A ones.

The fact that similar phenomena are observed in such diverse materials as glass (Sommer, 1969), alumina (Suresh and Tschegg, 1987), steels (Hourlier and Pineau, 1979; Yates and Miller, 1989; Lazarus, 1997), rocks (Pollard et al., 1982; Pollard and Aydin, 1988; Cooke and Pollard, 1996), PMMA (Lazarus et al., 2008), gypsum and cheese (Goldstein and Osipenko, 2012) strongly suggests that the microstructure of these materials plays little or no role and that the standard tools of macroscopic Linear Elastic Fracture Mechanics (LEFM) should be able to explain the observations made.

The problem was recently attacked by Pons and Karma (2010) from a completely different, numerical angle. These authors performed numerical simulations of crack propagation in mode I+III using a "phase field" model previously developed by Karma et al. (2001), which included a heuristic description of short scale failure in the process zone around the crack front. These simulations reproduced many of the experimental observations made, including the tendency of the crack to deviate out of its original plane through formation of an array of inclined facets, and the tendency of type A facets to propagate ahead of type B ones.

The relationship of Karma et al. (2001)'s phase-field model to conventional LEFM has been examined by Hakim and Karma (2009). These authors showed that, in the asymptotic limit of this model where the system size is large compared to the process zone size, quasistatic propagation of a crack in some isotropic medium is governed by a combination 
of two classical LEFM criteria, applied all along the crack front: (i) a condition of uniform energy-release-rate (Griffith (1920)'s criterion); and (ii) a condition of zero SIF of mode II (Goldstein and Salganik (1974)'s principle of local symmetry). This conclusion, combined with the success of the phase-field model in the description of crack propagation in mode I+III evidenced by Pons and Karma (2010), again suggests that the problem should be tractable within the framework of standard LEFM.

The concepts and tools of LEFM have indeed been used in a few theoretical approaches of the topic. In the case of abrupt, discontinuous tilting of the facets, Pollard et al. (1982) suggested to estimate the "tilt angle", that is the angle made by type A facets with the original crack plane, by (i) calculating the traction vector acting on each possible future infinitesimal facet by using the asymptotic expression of the stresses near the original crack front, and (ii) assuming the shear component of this traction vector to be zero on that facet which will actually develop. This led to the following expression of the tilt angle $\alpha$ :

$$
\alpha=\frac{1}{2} \arctan \left(\frac{K_{I I I}^{3 D} / K_{I}^{3 D}}{1 / 2-\nu}\right)
$$

where $\nu$ denotes Poisson's ratio and $K_{I}^{3 D}$ and $K_{I I I}^{3 D}$ the mode I and III SIF along the initial, straight crack front. (The notations $K_{I}^{3 D}, K_{I I I}^{3 D}$ for these SIF are used in order to distinguish them from the 2D SIF $K_{I}, K_{I I}$ introduced below). Formula (1) was shown by Lazarus et al. (2001b), on the basis of experiments performed on steels, to somewhat overestimate the tilt angle.

Lazarus et al. (2001b) also showed, using theoretical estimates of the SIF after some short continuous twisting (Lazarus et al., 2001a), that the energy-release-rate is larger at the center of type A facets than at the center of type B ones. This implies that propagation of type A facets is "energetically favored" with respect to that of type B ones, which provides a theoretical basis to Hourlier and Pineau (1979)'s observation that the length of type A facets is larger than that of type B ones.

More recently, using previous results of Gao and Rice (1986) and Movchan et al. (1998) on in-plane and out-of-plane perturbations of a plane crack, Leblond et al. (2011) performed, apparently for the first time, a rigorous theoretical analysis of the possible bifurcation from coplanar to non-coplanar crack propagation in mode I+III; this analysis was based, in line with Hakim and Karma (2009)'s and Pons and Karma (2010)'s findings reported above, on the assumption of a constant value of the local energy-release-rate and a zero value of the local SIF of mode II all along the crack front. Such a bifurcation was concluded to exist for values of the ratio $K_{I I I}^{3 D} / K_{I}^{3 D}$ larger than some threshold depending on Poisson's ratio.

This analysis however left several questions open. First, since the threshold was found to be of the order of 0.5 for standard values of $\nu$, it could not explain the fact that deviations of the crack from its original plane are currently observed for much smaller values of $K_{I I I}^{3 D} / K_{I}^{3 D}$ - possible answers to this issue will be proposed in some future papers. Second, it could not provide an expression similar to Pollard et al. (1982)'s formula (1) for the tilt angle of incipient, abruptly tilting type A facets, since it was based on the assumption of gradual twisting of these facets. Third, it could not provide a formula for the stationary tilt angle of well-developed type A facets either, since as a consequence of 
the linear perturbation approach adopted, the deviation of the crack from coplanarity and the resulting tilt angle were predicted to increase exponentially, and therefore indefinitely with the distance of propagation.

The purpose of this paper is precisely to lay the grounds for future predictions of the stationary tilt angle of long type A facets, assuming complete absence of type B facets. Unlike in the case of incipient facets, the local stress field near the lateral fronts of welldeveloped facets is no longer influenced by the initial crack, and the problem becomes essentially 2D in a plane perpendicular to the main direction of propagation. The situation is that of an infinite plate containing an infinite and periodic array of identical finite cracks inclined at some angle $\alpha$ on a straight line, and subjected to uniform remote stresses. To predict the stationary tilt angle, the first step consists in solving the $2 \mathrm{D}$ elasticity problem thus posed, so as to get formulae for the $2 \mathrm{D}$ SIF $K_{I}, K_{I I}$ on the lateral sides of the facets in terms of the angle $\alpha$ and the remote stresses. In a second step, these formulae may be combined with some appropriate propagation criterion to get an expression of $\alpha$.

The paper is organized as follows:

- In Section 2, we present the 2D elasticity problem to be solved and the notations used.

- In Section 3, as a prerequisite, we derive formulae for the first three terms of the asymptotic expansion of Muskhelishvili (1953)'s complex potentials near the tip of an arbitrarily oriented crack. Assuming then the crack to make a small angle $\alpha$ with the horizontal axis, we obtain from there expressions of these first three terms accurate to first order in $\alpha$.

- In Section 4, we briefly recall the analytical solution of the problem at order 0 in $\alpha$, identical to Westergaard (1939)'s and Koiter (1959)'s solution for an infinite array of cracks aligned on a straight line.

- In Section 5, using the results of the two preceding sections, we derive the analytical solution of the problem at order 1 in $\alpha$.

- In Section 6, we extend the first-order solution in an approximate way to larger values of $\alpha$, by using the well-known solution for a unique crack arbitrarily inclined over the horizontal axis as an extra reference.

- In Section 7, the approximate expressions of the 2D SIF $K_{I}, K_{I I}$ thus defined are assessed through comparison with the results of some finite element computations.

- In Section 8, as a first, tentative example of application of these formulae, we combine them with the heuristic hypothesis that the facets propagate laterally under conditions of vanishing $K_{I I}$, to get an approximate prediction of the tilt angle $\alpha$.

\section{Statement of the problem - Notations}

We consider (Figure 1) an infinite plate made of some homogeneous and isotropic elastic material and containing an infinite and periodic array of identical cracks. The centers of these cracks are aligned on the horizontal axis $O x_{1}$ but the cracks, instead of being collinear to this axis, are inclined at an angle $\alpha$ over it. The common projected length of the cracks onto the axis $O x_{1}$ is denoted $2 c$, and the distance between their successive 
centers (the period of the periodic geometry) is denoted $2 d$; $c$ is assumed to be smaller than $d$. The plate is loaded in conditions of plane strain, generalized plane strain or plane stress ${ }^{1}$ through uniform remote stresses $\sigma_{11}^{\infty}, \sigma_{22}^{\infty}, \sigma_{12}^{\infty}$, and the cracks are traction-free. There are no body forces.

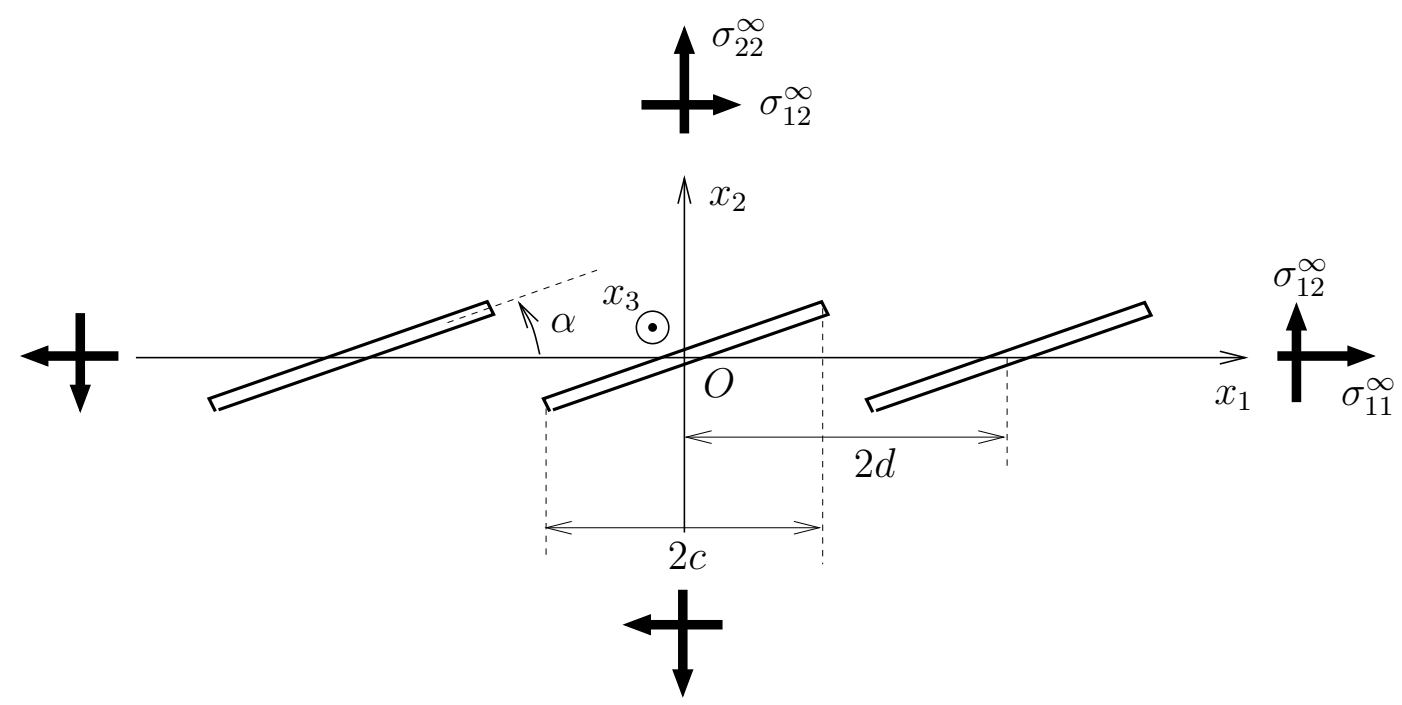

Fig. 1. An infinite periodic array of inclined cracks

This model 2D cracked geometry is related to the original 3D geometry resulting from non-coplanar propagation of a crack loaded in mode I+III, in the absence of type B facets, in the following way. It represents a section of this 3D geometry by a plane perpendicular to the main direction of crack propagation, and located far enough from the original crack front, in this direction, for type A facets to have reached a stationary tilt angle $\alpha$. The axis $O x_{1}$ is parallel to the original crack front, the axis $O x_{2}$ perpendicular to the original crack plane, and the axis $O x_{3}$ parallel to the main direction of propagation. The $2 \mathrm{D}$ cracks represent the intersections of the type A facets and the plane $O x_{1} x_{2}$, and their tips are located on the lateral sides of these facets.

The problem will be solved analytically using Muskhelishvili (1953)'s method up to first order in the angle $\alpha$, considered as small, and numerically by the finite element method, for arbitrary values of $\alpha$.

Muskhelishvili (1953)'s method basically consists in looking for the expressions of two complex analytic functions $\Phi(z), \Psi(z)$ of the complex variable $z \equiv x_{1}+i x_{2}$, which together define the stress field. However it will be more convenient in the present case to use, instead of the function $\Psi(z)$, the function (Muskhelishvili (1953), p. 495)

$$
\Omega(z) \equiv \bar{\Phi}(z)+z \overline{\Phi^{\prime}}(z)+\bar{\Psi}(z) \quad(\text { where } \bar{f}(z) \equiv \overline{f(\bar{z})})
$$

analytic on the conjugate of the domain of definition of $\Phi(z)$ and $\Psi(z)$. The relations connecting the stresses to the "potentials" $\Phi(z)$ and $\Omega(z)$ read (Muskhelishvili (1953),

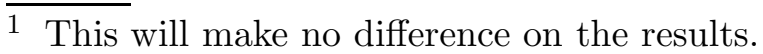


pp. 114 and 495)

$$
\begin{cases}\sigma_{11}+\sigma_{22} & =4 \operatorname{Re}[\Phi(z)]=2[\Phi(z)+\overline{\Phi(z)}] \\ \sigma_{22}-\sigma_{11}+2 i \sigma_{12} & =2\left[\bar{\Omega}(z)-\Phi(z)+(\bar{z}-z) \Phi^{\prime}(z)\right] .\end{cases}
$$

These relations permit to write the conditions on $\Phi(z)$ and $\Omega(z)$ resulting from the boundary conditions on the crack lips and at infinity.

We shall look for the expressions of the functions appearing in the first-order expansion of $\Phi(z)$ and $\Omega(z)$ :

$$
\Phi(z) \equiv \Phi^{0}(z)+\alpha \Phi^{1}(z)+O\left(\alpha^{2}\right) \quad ; \quad \Omega(z) \equiv \Omega^{0}(z)+\alpha \Omega^{1}(z)+O\left(\alpha^{2}\right) .
$$

\section{Asymptotic expressions of Muskhelishvili's potentials near a crack tip}

The first task is to derive the asymptotic expressions of Muskhelishvili's potentials $\Phi(z)$ and $\Omega(z)$ near the tips of a crack in terms of the various constants appearing in the expansion of the stresses. This will be done first for an arbitrarily oriented crack, then for a crack making a small angle with the horizontal axis $O x_{1}$, to first order in this angle.

\subsection{Case of an arbitrarily oriented crack}

We thus consider first a crack with arbitrary tip $z_{0}$, making an arbitrary angle $\theta_{0}$ with the axis $O x_{1}$ (Figure 2). Use is made of the "naturally associated" polar coordinates $r, \theta$ defined by $z-z_{0} \equiv r e^{i\left(\theta_{0}+\theta\right)}, r>0,-\pi<\theta<\pi, z$ denoting the current point in the complex plane.

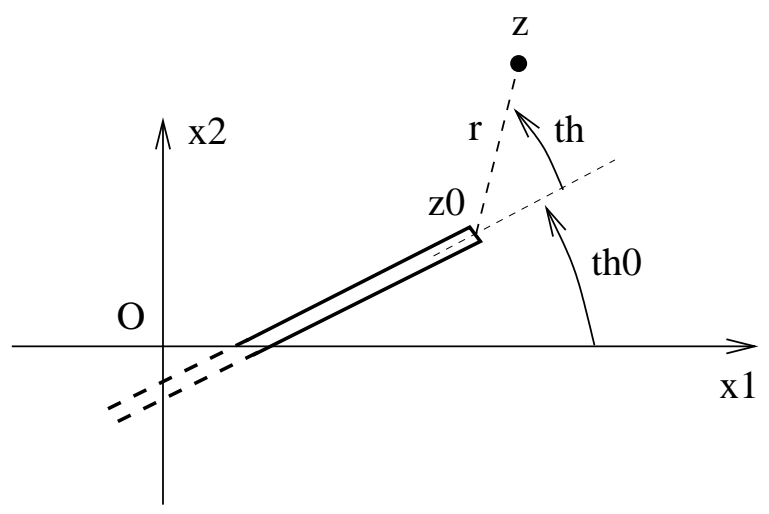

Fig. 2. The vicinity of the tip of an inclined crack

To first determine the asymptotic form of the potential $\Phi(z)$, one must consider successively the first three terms of the stress expansion:

- For the first term proportional to $r^{-1 / 2}$, Williams's classical formulae imply that $\sigma_{11}+$ $\sigma_{22}=\sigma_{r r}+\sigma_{\theta \theta}=\frac{2}{\sqrt{2 \pi r}} \operatorname{Re}\left[\left(K_{I}-i K_{I I}\right) e^{-i \theta / 2}\right]$ where $K_{I}$ and $K_{I I}$ denote the SIF. By 
equation $(3)_{1}$, this expression may be matched by taking a potential $\Phi(z)$ equal to $\frac{\left(K_{I}-i K_{I I}\right) e^{i \theta_{0} / 2}}{2 \sqrt{2 \pi\left(z-z_{0}\right)}}$.

- For the second term proportional to $r^{0}=1, \sigma_{11}+\sigma_{22}=\sigma_{r r}+\sigma_{\theta \theta}=T$ where $T$ denotes the non-singular stress, representing a uniform uniaxial stress field parallel to the crack. This expression corresponds to a potential $\Phi(z)$ equal to $\frac{T}{4}+i \gamma$ for some real coefficient $\gamma \cdot{ }^{2}$

- Finally the third term proportional to $r^{1 / 2}$ must correspond to a potential $\Phi(z)$ of the form $B e^{-i \theta_{0} / 2} \sqrt{\frac{z-z_{0}}{2 \pi}}$ for some complex coefficient $B$.

Gathering these elements, one concludes that $\Phi(z)$ is asymptotically of the form

$$
\Phi(z)=\frac{K e^{i \theta_{0} / 2}}{2 \sqrt{2 \pi\left(z-z_{0}\right)}}+\frac{T}{4}+i \gamma+B e^{-i \theta_{0} / 2} \sqrt{\frac{z-z_{0}}{2 \pi}}+O\left(z-z_{0}\right) \quad \text { for } z \rightarrow z_{0}
$$

where

$$
K \equiv K_{I}-i K_{I I}
$$

is the complex SIF.

To now determine the asymptotic form of the potential $\Omega(z)$, or rather $\bar{\Omega}(z)$, one must consider the quantity $\sigma_{22}-\sigma_{11}+2 i \sigma_{12}=\left(\sigma_{\theta \theta}-\sigma_{r r}+2 i \sigma_{r \theta}\right) e^{-2 i\left(\theta_{0}+\theta\right)}$, the asymptotic expression of which is, by Williams's classical formulae, $\frac{e^{-2 i \theta_{0}}}{2 \sqrt{2 \pi r}}\left[\left(-K_{I}+i K_{I I}\right) e^{-5 i \theta / 2}+\left(K_{I}+3 i K_{I I}\right) e^{-i \theta / 2}\right]$. Using this expression, equation $(3)_{2}$ and the asymptotic form (5) of $\Phi(z)$, one gets after a tedious but straightforward calculation the following asymptotic expression of $\bar{\Omega}(z)$ :

$$
\begin{aligned}
\bar{\Omega}(z)= & -\frac{i K e^{i \theta_{0} / 2} \operatorname{Im} z_{0}}{2 \sqrt{2 \pi}\left(z-z_{0}\right)^{3 / 2}} \\
& +\frac{K e^{i \theta_{0} / 2}+\left(K_{I}+3 i K_{I I}\right) e^{-3 i \theta_{0} / 2}+4 i B e^{-i \theta_{0} / 2} \operatorname{Im} z_{0}}{4 \sqrt{2 \pi\left(z-z_{0}\right)}}+O(1) \quad \text { for } z \rightarrow z_{0}
\end{aligned}
$$

Of course, the cut of the power functions in equations (5) and (7) is along the crack; that is, they are defined with $-\pi+\theta_{0}<\arg \left(z-z_{0}\right)<\pi+\theta_{0}$.

\subsection{Case of a crack slightly inclined over the horizontal axis}

We now consider the case of a crack making a small angle $\alpha$ with the axis $O x_{1}$ (Figure 3 ). The projections of the crack tips onto this axis are denoted $a$ and $b$ respectively.

We wish to find the asymptotic expressions of the functions $\Phi^{0}(z), \Phi^{1}(z), \Omega^{0}(z), \Omega^{1}(z)$ appearing in the first-order expansions (4) of the potentials $\Phi(z)$ and $\Omega(z)$ in powers of $\alpha$. This may be done by looking for the expansions of the asymptotic expressions (5), (7) of these potentials. It is necessary here to distinguish between the left and right tips.

$\overline{2}$ A special notation is used for this coefficient in order to emphasize that is has no influence whatsoever on the stresses, see equations (3). 


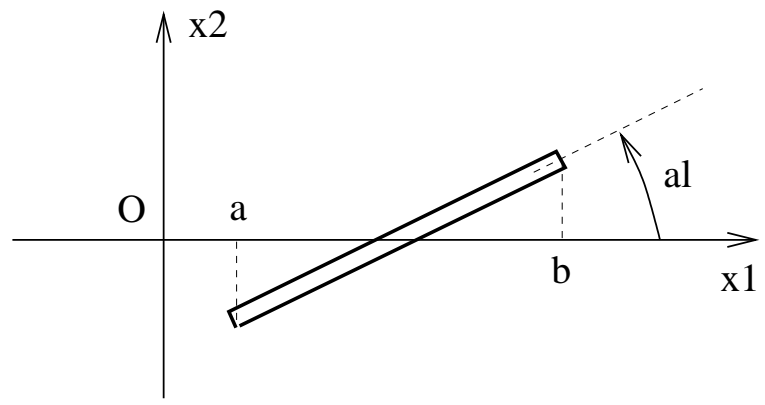

Fig. 3. A crack inclined by a small angle on the horizontal axis

- The position of the right tip and the inclination of the crack there are given by $z_{0} \equiv$ $b+i \alpha \frac{b-a}{2}+O\left(\alpha^{2}\right), \theta_{0} \equiv \alpha$. Using these formulae, the first-order expressions of the coefficients $K_{b}, T_{b}, \gamma_{b}, B_{b}$ of the stress expansion near this tip,

$$
\left\{\begin{array}{l}
K_{b} \equiv K_{b}^{0}+\alpha K_{b}^{1}+O\left(\alpha^{2}\right) \\
T_{b} \equiv T_{b}^{0}+\alpha T_{b}^{1}+O\left(\alpha^{2}\right) \\
\gamma_{b} \equiv \gamma_{b}^{0}+\alpha \gamma_{b}^{1}+O\left(\alpha^{2}\right) \\
B_{b} \equiv B_{b}^{0}+\alpha B_{b}^{1}+O\left(\alpha^{2}\right)
\end{array}\right.
$$

and expanding equation (5) and the conjugate of equation (7) to first order in $\alpha$, one gets the following results after some calculations: at order 0 ,

$$
\left\{\begin{array}{l}
\Phi^{0}(z)=\frac{K_{b}^{0}}{2[2 \pi(z-b)]_{-}^{1 / 2}}+\frac{T_{b}^{0}}{4}+i \gamma_{b}^{0}+B_{b}^{0}\left(\frac{z-b}{2 \pi}\right)_{-}^{1 / 2}+O(z-b) \\
\Omega^{0}(z)=\frac{K_{b}^{0}}{2[2 \pi(z-b)]_{-}^{1 / 2}}+O(1)
\end{array} \quad \text { for } z \rightarrow b,\right.
$$

and at order 1 ,

$$
\left\{\begin{array}{l}
\Phi^{1}(z)=\frac{i(b-a) K_{b}^{0}}{8 \sqrt{2 \pi}(z-b)_{-}^{3 / 2}}+\frac{2 K_{b}^{1}+i K_{b}^{0}-i(b-a) B_{b}^{0}}{4[2 \pi(z-b)]_{-}^{1 / 2}}+O(1) \\
\Omega^{1}(z)=\frac{(b-a)\left(i K_{b I}^{0}-3 K_{b I I}^{0}\right)}{8 \sqrt{2 \pi}(z-b)_{-}^{3 / 2}}+O\left[(z-b)^{-1 / 2}\right]
\end{array} \quad \text { for } z \rightarrow b .\right.
$$

In these expressions the lower index _ in the power functions indicates that their cut is along the left horizontal half-line originating from the point $b$; that is, they are defined with $-\pi<\arg (z-b)<\pi$. Note that the only "first-order coefficient" appearing in equations (10) is $K_{b}^{1}$, the other coefficients $T_{b}^{1}, \gamma_{b}^{1}, B_{b}^{1}$ being absent.

- Similarly, the position of the left tip and the inclination of the crack there are given by $z_{0} \equiv a-i \alpha \frac{b-a}{2}+O\left(\alpha^{2}\right), \theta_{0} \equiv \pi+\alpha$. Using these expressions and writing the coefficients 
$K_{a}, T_{a}, \gamma_{a}, B_{a}$ of the stress expansion near this tip in the form

$$
\left\{\begin{aligned}
K_{a} & \equiv K_{a}^{0}+\alpha K_{a}^{1}+O\left(\alpha^{2}\right) \\
T_{a} & \equiv T_{a}^{0}+\alpha T_{a}^{1}+O\left(\alpha^{2}\right) \\
\gamma_{a} & \equiv \gamma_{a}^{0}+\alpha \gamma_{a}^{1}+O\left(\alpha^{2}\right) \\
B_{a} & \equiv B_{a}^{0}+\alpha B_{a}^{1}+O\left(\alpha^{2}\right)
\end{aligned}\right.
$$

one gets from equations (5) and (7):

$$
\left\{\begin{array}{l}
\Phi^{0}(z)=\frac{i K_{a}^{0}}{2[2 \pi(z-a)]_{+}^{1 / 2}}+\frac{T_{a}^{0}}{4}+i \gamma_{a}^{0}-i B_{a}^{0}\left(\frac{z-a}{2 \pi}\right)_{+}^{1 / 2}+O(z-a) \\
\Omega^{0}(z)=\frac{i K_{a}^{0}}{2[2 \pi(z-a)]_{+}^{1 / 2}}+O(1)
\end{array}\right.
$$

and

$$
\left\{\begin{array}{l}
\Phi^{1}(z)=\frac{(b-a) K_{a}^{0}}{8 \sqrt{2 \pi}(z-a)_{+}^{3 / 2}}+\frac{2 i K_{a}^{1}-K_{a}^{0}+(b-a) B_{a}^{0}}{4[2 \pi(z-a)]_{+}^{1 / 2}}+O(1) \\
\Omega^{1}(z)=\frac{(b-a)\left(K_{a I}^{0}+3 i K_{a I I}^{0}\right)}{8 \sqrt{2 \pi}(z-a)_{+}^{3 / 2}}+O\left[(z-a)^{-1 / 2}\right]
\end{array} \quad \text { for } z \rightarrow a\right.
$$

where the lower index + in the power functions indicates that their cut is along the right horizontal half-line originating from the point $a$; that is, they are defined with $0<\arg (z-a)<2 \pi$. Again, the sole first-order coefficient in equations (13) is $K_{a}^{1}$.

It may be observed that the first-order potentials $\Phi^{1}(z), \Omega^{1}(z)$ have stronger singularities than the zeroth-order potentials $\Phi^{0}(z), \Omega^{0}(z)$ near the points $a$ and $b$, since they behave like $(z-a)^{-3 / 2}$ and $(z-b)^{-3 / 2}$ there instead of $(z-a)^{-1 / 2}$ and $(z-b)^{-1 / 2}$. This is because the derivation of the asymptotic expressions (10) and (13) of $\Phi^{1}(z)$ and $\Omega^{1}(z)$ involves a differentiation of those, $(5)$ and $(7)$, of $\Phi(z)$ and $\bar{\Omega}(z)$ with respect to the position $z_{0}$ of the crack tip. The strong singularity of $\Phi^{1}(z)$ and $\Omega^{1}(z)$ will have an important impact upon the procedure of solution of the problem at order 1 .

\section{Zeroth-order solution}

The problem defined in Section 2, at order 0, that is for a zero tilt angle $\alpha$, is that of an infinite plate containing an infinite and periodic array of aligned cracks. The solution to this classical problem was provided by Westergaard (1939) and Koiter (1959) by different methods, and compiled in Tada et al. (2000)'s handbook. For completeness and as a guide to the solution of the first-order problem, we briefly recall here (with some slight improvements) Koiter (1959)'s treatment, based on Muskhelishvili (1953)'s method. 


\subsection{Determination of Muskhelishvili's potentials $\Phi^{0}(z)$ and $\Omega^{0}(z)$}

The determination of $\Phi^{0}(z)$ and $\Omega^{0}(z)$ uses the boundary conditions satisfied by these potentials on the crack lips and at infinity. ${ }^{3}$ On the crack lips, combination of equations (3) and the conditions of zero traction satisfied by the stress tensor $\boldsymbol{\sigma}^{0}$ yields

$$
\left(\sigma_{22}^{0}-i \sigma_{12}^{0}\right)\left(t^{ \pm}\right)=\Phi^{0}\left(t^{ \pm}\right)+\Omega^{0}\left(t^{\mp}\right)=0 \quad \text { for } t \in(2 n d-c, 2 n d+c), n \in \mathbb{Z}
$$

where $f\left(t^{ \pm}\right)$denotes the limit of $f(t \pm i \epsilon)$ for $\epsilon>0, \epsilon \rightarrow 0$. Taking the sum and the difference of these equations, one gets

$$
\left\{\begin{array}{l}
\left(\Phi^{0}+\Omega^{0}\right)\left(t^{+}\right)+\left(\Phi^{0}+\Omega^{0}\right)\left(t^{-}\right)=0 \\
\left(\Phi^{0}-\Omega^{0}\right)\left(t^{+}\right)-\left(\Phi^{0}-\Omega^{0}\right)\left(t^{-}\right)=0
\end{array} \quad \text { for } t \in(2 n d-c, 2 n d+c), n \in \mathbb{Z} .\right.
$$

At infinity, the following conditions must be met (Muskhelishvili (1953), pp. 494 and 495):

$$
\left\{\begin{array} { l } 
{ \Phi ^ { 0 } ( z ) = \Gamma + O ( \frac { 1 } { z ^ { 2 } } ) } \\
{ \Omega ^ { 0 } ( z ) = \Gamma + \overline { \Gamma ^ { \prime } } + O ( \frac { 1 } { z ^ { 2 } } ) }
\end{array} \quad \text { for } z \rightarrow \infty , \text { where } \quad \left\{\begin{array}{l}
\Gamma \equiv \frac{1}{4}\left(\sigma_{11}^{\infty}+\sigma_{22}^{\infty}\right) \\
\Gamma^{\prime} \equiv \frac{1}{2}\left(\sigma_{22}^{\infty}-\sigma_{11}^{\infty}\right)+i \sigma_{12}^{\infty}
\end{array}\right.\right.
$$

Let us first consider equation $(14)_{2}$, the easier to solve. The function $\left(\Phi^{0}-\Omega^{0}\right)(z)$ is a priori defined and analytic over the whole complex plane except on the real intervals $[2 n d-c, 2 n d+c], n \in \mathbb{Z}$. However it is continuous across the intervals $(2 n d-c, 2 n d+c)$ by equation $(14)_{2}$. By a well-known theorem of complex analysis, it follows that it is analytic everywhere except perhaps at the points $2 n d \pm c, n \in \mathbb{Z}$. But, by equations (9) and (12) (with $a \equiv 2 n d-c, b \equiv 2 n d+c$ ), it diverges less quickly than $(z-2 n d \mp c)^{-1}$ near these points. Hence its possible singularities at these points are removable, so that it is in fact analytic over the whole complex plane; that is, it is an entire function. Now it is bounded at infinity by equations $(15)_{1,2}$. Hence, by Liouville's theorem, it must be a constant, and the value of this constant is $-\overline{\Gamma^{\prime}}$ by equations $(15)_{1,2}$. Thus,

$$
\left(\Phi^{0}-\Omega^{0}\right)(z)=-\overline{\Gamma^{\prime}} \text { for all } z \in \mathbb{C} .
$$

In order to now determine the function $\left(\Phi^{0}+\Omega^{0}\right)(z)$, consider the auxiliary function

$$
\begin{aligned}
X(z) & \equiv\left[\sin \left(\frac{\pi z}{2 d}\right)+\sin \left(\frac{\pi c}{2 d}\right)\right]_{-}^{1 / 2}\left[\sin \left(\frac{\pi z}{2 d}\right)-\sin \left(\frac{\pi c}{2 d}\right)\right]_{-}^{1 / 2} \\
& \equiv\left[\sin ^{2}\left(\frac{\pi z}{2 d}\right)-\sin ^{2}\left(\frac{\pi c}{2 d}\right)\right]^{1 / 2}
\end{aligned}
$$

where the lower index _ in the power functions again indicates that their cut is along the left horizontal half-line originating from the points $\mp \sin \left(\frac{\pi c}{2 d}\right)$; that is, they are defined

3 Since the perturbation induced by the cracks extends indefinitely toward the left and the right, the expressions "at infinity", "when $z$ goes to infinity", etc. must be understood in the whole paper in the restrictive sense that $\operatorname{Im} z$ goes to $\pm \infty$ while $\operatorname{Re} z$ remains fixed. 
with $-\pi<\arg \left[\sin \left(\frac{\pi z}{2 d}\right) \pm \sin \left(\frac{\pi c}{2 d}\right)\right]<\pi$. This function possesses the following properties:

- If $z \in(2 n d-c, 2 n d+c), n \in \mathbb{Z}$, one of the quantities $\sin \left(\frac{\pi z}{2 d}\right)+\sin \left(\frac{\pi c}{2 d}\right), \sin \left(\frac{\pi z}{2 d}\right)-\sin \left(\frac{\pi c}{2 d}\right)$ is positive while the other is negative. Hence, when $z \in \mathbb{C}$ crosses the real interval $(2 n d-c, 2 n d+c)$, one of the square roots $\left[\sin \left(\frac{\pi z}{2 d}\right)+\sin \left(\frac{\pi c}{2 d}\right)\right]_{-}^{1 / 2},\left[\sin \left(\frac{\pi z}{2 d}\right)-\sin \left(\frac{\pi c}{2 d}\right)\right]_{-}^{1 / 2}$ changes sign while the other remains unchanged, so that $X(z)$ changes sign:

$$
X\left(t^{-}\right)=-X\left(t^{+}\right) \quad \text { for } t \in(2 n d-c, 2 n d+c), n \in \mathbb{Z}
$$

- If $z$ is real but lies outside of the intervals $[2 n d-c, 2 n d+c], n \in \mathbb{Z}$, the quantities $\sin \left(\frac{\pi z}{2 d}\right)+\sin \left(\frac{\pi c}{2 d}\right), \sin \left(\frac{\pi z}{2 d}\right)-\sin \left(\frac{\pi c}{2 d}\right)$ have identical signs. Hence when $z \in \mathbb{C}$ crosses the real line there, the square roots $\left[\sin \left(\frac{\pi z}{2 d}\right)+\sin \left(\frac{\pi c}{2 d}\right)\right]_{-}^{1 / 2},\left[\sin \left(\frac{\pi z}{2 d}\right)-\sin \left(\frac{\pi c}{2 d}\right)\right]_{-}^{1 / 2}$ remain both unchanged or change sign simultaneously, so that $X(z)$ remains unchanged. Thus this function is continuous across the real line except on the intervals [2nd $-c, 2 n d+c]$, $n \in \mathbb{Z}$. It is therefore analytic on the whole complex plane except on these intervals.

- Obviously,

$$
X(z) \sim \sin \left(\frac{\pi z}{2 d}\right) \quad(\rightarrow \infty) \text { for } z \rightarrow \infty
$$

- Obviously again, $X(z)$ is periodic of period $4 d$.

- When $z$ increases by $2 d$, the arguments $\left(\theta_{1}, \theta_{2}\right)$ of $\sin \left(\frac{\pi z}{2 d}\right)+\sin \left(\frac{\pi c}{2 d}\right), \sin \left(\frac{\pi z}{2 d}\right)-\sin \left(\frac{\pi c}{2 d}\right)$ become $\left(\theta_{2}-\pi, \theta_{1}-\pi\right)$ or $\left(\theta_{2}+\pi, \theta_{1}+\pi\right)$, depending on whether $z$ lies in the upper or lower half-plane; in both cases $X(z)$ changes sign. The function $X(z)$ is thus antiperiodic of antiperiod $2 d$ :

$$
X(z+2 d)=-X(z)
$$

Consider now the function $\left(\Phi^{0}+\Omega^{0}\right)(z)$. Equation $(14)_{1}$ may be rewritten, thanks to equation (18), in the form

$$
X\left(t^{+}\right)\left(\Phi^{0}+\Omega^{0}\right)\left(t^{+}\right)-X\left(t^{-}\right)\left(\Phi^{0}+\Omega^{0}\right)\left(t^{-}\right)=0 \quad \text { for } t \in(2 n d-c, 2 n d+c), n \in \mathbb{Z} .
$$

Thus the function $X(z)\left(\Phi^{0}+\Omega^{0}\right)(z)$, which is a priori defined and analytic over the whole complex plane except on the real intervals $[2 n d-c, 2 n d+c], n \in \mathbb{Z}$, is continuous across the intervals $(2 n d-c, 2 n d+c)$ and therefore analytic everywhere except perhaps at the points $2 n d \pm c, n \in \mathbb{Z}$. Since, by equations (9) and (12) (with $a \equiv 2 n d-c, b \equiv 2 n d+c$ ) and the definition $(17)$ of $X(z)$, it remains finite at these points, it is in fact analytic over the whole complex plane. Hence

$$
\left(\Phi^{0}+\Omega^{0}\right)(z) \equiv \frac{P(z)}{X(z)} \quad \text { for all } z \in \mathbb{C}
$$

where $P(z)$ is an entire function.

In order to determine the expression of $P(z)$, note that since $X(z)$ and $\left(\Phi^{0}+\Omega^{0}\right)(z)$ are periodic of periods $4 d$ and $2 d$ respectively, and $X(z)$ antiperiodic of antiperiod $2 d, P(z)$ must be periodic of period $4 d$ and antiperiodic of antiperiod $2 d$. The first property implies that it must be of the form 


$$
P(z) \equiv A_{0}+\sum_{n=1}^{+\infty}\left[A_{n} \cos \left(\frac{n \pi z}{2 d}\right)+B_{n} \sin \left(\frac{n \pi z}{2 d}\right)\right]
$$

for some complex constants $A_{0}, A_{1}, B_{1}, A_{2}, B_{2}, \ldots$, and the second that all constants of even index are necessarily zero; thus it must be of the form

$$
P(z)=\sum_{n=0}^{+\infty}\left\{A_{2 n+1} \cos \left[\frac{(2 n+1) \pi z}{2 d}\right]+B_{2 n+1} \sin \left[\frac{(2 n+1) \pi z}{2 d}\right]\right\} .
$$

In particular,

$$
P\left(i x_{2}\right)=\sum_{n=0}^{+\infty}\left\{A_{2 n+1} \cosh \left[\frac{(2 n+1) \pi x_{2}}{2 d}\right]+i B_{2 n+1} \sinh \left[\frac{(2 n+1) \pi x_{2}}{2 d}\right]\right\}
$$

so that, by equation (19),

$$
\begin{aligned}
\left(\Phi^{0}+\Omega^{0}\right)\left(i x_{2}\right)=\frac{P\left(i x_{2}\right)}{X\left(i x_{2}\right)} \sim \sum_{n=0}^{+\infty}\{ & -i A_{2 n+1} \frac{\cosh \left[\frac{(2 n+1) \pi x_{2}}{2 d}\right]}{\sinh \left(\frac{\pi x_{2}}{2 d}\right)} \\
& \left.+B_{2 n+1} \frac{\sinh \left[\frac{(2 n+1) \pi x_{2}}{2 d}\right]}{\sinh \left(\frac{\pi x_{2}}{2 d}\right)}\right\} \text { for } x_{2} \rightarrow \pm \infty .
\end{aligned}
$$

But $\left(\Phi^{0}+\Omega^{0}\right)\left(i x_{2}\right)$ must remain finite for $x_{2} \rightarrow \pm \infty$ by equations $(15)_{1,2}$. Now all terms $\cosh \left[\frac{(2 n+1) \pi x_{2}}{2 d}\right] / \sinh \left(\frac{\pi x_{2}}{2 d}\right), \sinh \left[\frac{(2 n+1) \pi x_{2}}{2 d}\right] / \sinh \left(\frac{\pi x_{2}}{2 d}\right), n \geq 1$, are linearly independent and diverge for $x_{2} \rightarrow \pm \infty$; hence their coefficients must be zero, that is, only $A_{1}$ and $B_{1}$ may be nonzero. Then

$$
\left(\Phi^{0}+\Omega^{0}\right)\left(i x_{2}\right) \rightarrow \mp i A_{1}+B_{1} \quad \text { for } x_{2} \rightarrow \pm \infty .
$$

But both of these limits must be equal to $2 \Gamma+\overline{\Gamma^{\prime}}$ by equations $(15)_{1,2}$; this implies that

$$
A_{1}=0 \quad ; \quad B_{1}=2 \Gamma+\overline{\Gamma^{\prime}}
$$

so that, finally,

$$
\left(\Phi^{0}+\Omega^{0}\right)(z)=\left(2 \Gamma+\overline{\Gamma^{\prime}}\right) \frac{\sin \left(\frac{\pi z}{2 d}\right)}{\left[\sin ^{2}\left(\frac{\pi z}{2 d}\right)-\sin ^{2}\left(\frac{\pi c}{2 d}\right)\right]^{1 / 2}} \quad \text { for all } z \in \mathbb{C} .
$$

Combining equations (16) and (22) and the expressions $(15)_{3,4}$ of the constants $\Gamma$ and $\Gamma^{\prime}$, one gets the final expressions of $\Phi^{0}(z)$ and $\Omega^{0}(z)$ (Koiter, 1959):

$$
\left\{\begin{array}{l}
\Phi^{0}(z)=\frac{1}{2}\left(\sigma_{22}^{\infty}-i \sigma_{12}^{\infty}\right) \frac{\sin \left(\frac{\pi z}{2 d}\right)}{\left[\sin ^{2}\left(\frac{\pi z}{2 d}\right)-\sin ^{2}\left(\frac{\pi c}{2 d}\right)\right]^{1 / 2}}+\frac{1}{4}\left(\sigma_{11}^{\infty}-\sigma_{22}^{\infty}\right)+\frac{i}{2} \sigma_{12}^{\infty} \\
\Omega^{0}(z)=\frac{1}{2}\left(\sigma_{22}^{\infty}-i \sigma_{12}^{\infty}\right) \frac{\sin \left(\frac{\pi z}{2 d}\right)}{\left[\sin ^{2}\left(\frac{\pi z}{2 d}\right)-\sin ^{2}\left(\frac{\pi c}{2 d}\right)\right]^{1 / 2}}-\frac{1}{4}\left(\sigma_{11}^{\infty}-\sigma_{22}^{\infty}\right)-\frac{i}{2} \sigma_{12}^{\infty}
\end{array} \text { for all } z \in \mathbb{C} .\right.
$$


The SIF, non-singular stresses and coefficients $B$, being identical at all crack tips for obvious symmetry reasons, will be denoted simply $K_{I}^{0}, K_{I I}^{0}, T^{0}, B^{0}$, without a lower index ${ }_{a}$ or ${ }_{b}$ like in Subsection 3.2. They may be determined by considering points lying slightly ahead of the tip $c$, that is of the form $z=c+r, r>0, r \rightarrow 0$, expanding the exact expression $(23)_{1}$ of $\Phi^{0}(z=c+r)$ up to order $r^{1 / 2}$, and comparing the result to the asymptotic expression $(9)_{1}$ (with $b \equiv c$ ). One thus gets after some calculations:

$$
\left\{\begin{array}{l}
K_{I}^{0}=\sigma_{22}^{\infty} \sqrt{2 d \tan \left(\frac{\pi c}{2 d}\right)} \\
K_{I I}^{0}=\sigma_{12}^{\infty} \sqrt{2 d \tan \left(\frac{\pi c}{2 d}\right)} \\
T^{0}=\sigma_{11}^{\infty}-\sigma_{22}^{\infty} \\
B^{0}=\left(\sigma_{22}^{\infty}-i \sigma_{12}^{\infty}\right) \sqrt{2 d \tan \left(\frac{\pi c}{2 d}\right)}\left[\frac{\pi}{4 d} \cot \left(\frac{\pi c}{2 d}\right)-\frac{\pi}{8 d} \cot \left(\frac{\pi c}{d}\right)\right] .
\end{array}\right.
$$

The expressions of $K_{I}^{0}$ and $K_{I I}^{0}$ here may be found in Tada et al. (2000)'s handbook, although they were not given in the original sources (Westergaard, 1939; Koiter, 1959). The expressions of $T^{0}$ and $B^{0}$, on the other hand, seem to be new. The expression of the non-singular stress is surprisingly exactly the same as for a unique crack.

\section{$5 \quad$ First-order solution}

\subsection{Boundary conditions on the crack lips and at infinity}

To determine the potentials $\Phi^{1}(z), \Omega^{1}(z)$ of the first-order solution and the associated SIF $K_{I}^{1}, K_{I I}^{1}$, the first task is to write the boundary conditions satisfied by these potentials on the crack lips and at infinity. This will require using the first-order expansion of the stresses:

$$
\boldsymbol{\sigma} \equiv \boldsymbol{\sigma}^{0}+\alpha \boldsymbol{\sigma}^{1}+O\left(\alpha^{2}\right)
$$

Up to first order in $\alpha$, the coordinates of the current point on the $n$-th crack are $\left(x_{1}, x_{2}\right)=$ $(t, \alpha(t-2 n d) \pm \epsilon)$, where $t \in(2 n d-c, 2 n d+c), \epsilon>0, \epsilon \rightarrow 0$, and the normal vector to this crack is $\mathbf{e}_{2}-\alpha \mathbf{e}_{1}$ where $\mathbf{e}_{1}$ and $\mathbf{e}_{2}$ are the basis vectors associated to the coordinates $x_{1}, x_{2}$. Therefore the conditions of zero traction on the crack read, momentarily considering $\boldsymbol{\sigma}^{0}$ and $\boldsymbol{\sigma}^{1}$ as functions of the real variables $x_{1}$ and $x_{2}$ instead of the complex one $z \equiv x_{1}+i x_{2}$ :

$$
\begin{gathered}
\left(\boldsymbol{\sigma}^{0}+\alpha \boldsymbol{\sigma}^{1}\right)(t, \alpha(t-2 n d) \pm \epsilon) \cdot\left(\mathbf{e}_{2}-\alpha \mathbf{e}_{1}\right)+O\left(\alpha^{2}\right)=\mathbf{0} \\
\Leftrightarrow \boldsymbol{\sigma}^{0}(t, \pm \epsilon) \cdot \mathbf{e}_{2}+\alpha\left[\boldsymbol{\sigma}^{1}(t, \pm \epsilon) \cdot \mathbf{e}_{2}+(t-2 n d) \frac{\partial \boldsymbol{\sigma}^{0}}{\partial x_{2}}(t, \pm \epsilon) \cdot \mathbf{e}_{2}-\boldsymbol{\sigma}^{0}(t, \pm \epsilon) \cdot \mathbf{e}_{1}\right]+O\left(\alpha^{2}\right)=\mathbf{0} .
\end{gathered}
$$

At order 0 in $\alpha$, this condition gives of course $\boldsymbol{\sigma}^{0}(t, \pm \epsilon) \cdot \mathbf{e}_{2}=\mathbf{0}$. At order 1 , it gives 


$$
\begin{gathered}
\boldsymbol{\sigma}^{1}(t, \pm \epsilon) \cdot \mathbf{e}_{2}+(t-2 n d) \frac{\partial \boldsymbol{\sigma}^{0}}{\partial x_{2}}(t, \pm \epsilon) \cdot \mathbf{e}_{2}-\boldsymbol{\sigma}^{0}(t, \pm \epsilon) \cdot \mathbf{e}_{1}=\mathbf{0} \\
\Leftrightarrow \quad \sigma_{i 2}^{1}(t, \pm \epsilon)+(t-2 n d) \frac{\partial \sigma_{i 2}^{0}}{\partial x_{2}}(t, \pm \epsilon)-\sigma_{i 1}^{1}(t, \pm \epsilon)=0 \quad(i=1,2) .
\end{gathered}
$$

But $\frac{\partial \sigma_{i 2}^{0}}{\partial x_{2}}=-\frac{\partial \sigma_{i 1}^{0}}{\partial x_{1}}$ by the equilibrium equations. Hence this condition may be rewritten as

$$
\sigma_{i 2}^{1}(t, \pm \epsilon)=(t-2 n d) \frac{\partial \sigma_{i 1}^{0}}{\partial x_{1}}(t, \pm \epsilon)+\sigma_{i 1}^{1}(t, \pm \epsilon)=\frac{d}{d t}\left[(t-2 n d) \sigma_{i 1}^{0}(t, \pm \epsilon)\right] \quad(i=1,2)
$$

or equivalently since $\sigma_{21}^{0}(t, \pm \epsilon)=0$ on the cracks, considering again $\boldsymbol{\sigma}^{0}$ and $\boldsymbol{\sigma}^{1}$ as functions of the complex variable $x_{1}+i x_{2}$ :

$$
\left(\sigma_{22}^{1}-i \sigma_{12}^{1}\right)\left(t^{ \pm}\right)=-i \frac{d}{d t}\left[(t-2 n d) \sigma_{11}^{0}\left(t^{ \pm}\right)\right] \quad \text { for } t \in(2 n d-c, 2 n d+c), n \in \mathbb{Z}
$$

To express this condition in terms of the potentials $\Phi^{0}(z), \Omega^{0}(z), \Phi^{1}(z), \Omega^{1}(z)$, it suffices to note that on the cracks, by equations (3) and the boundary condition $\sigma_{22}^{0}\left(t^{ \pm}\right)=0$, $\left(\sigma_{22}^{1}-i \sigma_{12}^{1}\right)\left(t^{ \pm}\right)=\Phi^{1}\left(t^{ \pm}\right)+\Omega^{1}\left(t^{\mp}\right)$ and $\sigma_{11}^{0}\left(t^{ \pm}\right)=\sigma_{11}^{0}\left(t^{ \pm}\right)+\sigma_{22}^{0}\left(t^{ \pm}\right)=4 \operatorname{Re}\left[\Phi^{0}\left(t^{ \pm}\right)\right]$. Hence equation (26) may be rewritten in the form

$$
\Phi^{1}\left(t^{ \pm}\right)+\Omega^{1}\left(t^{\mp}\right)=-4 i \frac{d}{d t}\left\{(t-2 n d) \operatorname{Re}\left[\Phi^{0}\left(t^{ \pm}\right)\right]\right\} \quad \text { for } t \in(2 n d-c, 2 n d+c), n \in \mathbb{Z}
$$

or equivalently, taking the sum and the difference of these equations:

$$
\left\{\begin{aligned}
\left(\Phi^{1}+\right. & \left.\Omega^{1}\right)\left(t^{+}\right)+\left(\Phi^{1}+\Omega^{1}\right)\left(t^{-}\right) \\
& =-4 i \frac{d}{d t}\left\{(t-2 n d) \operatorname{Re}\left[\Phi^{0}\left(t^{+}\right)+\Phi^{0}\left(t^{-}\right)\right]\right\} \\
\left(\Phi^{1}-\right. & \left.\Omega^{1}\right)\left(t^{+}\right)-\left(\Phi^{1}-\Omega^{1}\right)\left(t^{-}\right) \\
& =-4 i \frac{d}{d t}\left\{(t-2 n d) \operatorname{Re}\left[\Phi^{0}\left(t^{+}\right)-\Phi^{0}\left(t^{-}\right)\right]\right\}
\end{aligned} \quad \text { for } t \in(2 n d-c, 2 n d+c), n \in \mathbb{Z} .\right.
$$

At infinity, since the stresses $\sigma_{11}^{\infty}, \sigma_{22}^{\infty}, \sigma_{12}^{\infty}$ resulting from the potentials $\Phi(z)=\Phi^{0}(z)+$ $\alpha \Phi^{1}(z)+O\left(\alpha^{2}\right), \Omega(z)=\Omega^{0}(z)+\alpha \Omega^{1}(z)+O\left(\alpha^{2}\right)$ are independent of $\alpha$, those resulting from the potentials $\Phi^{1}(z), \Omega^{1}(z)$ must be zero. The conditions to be satisfied by these potentials are therefore (Muskhelishvili (1953), pp. 494 and 495):

$$
\Phi^{1}(z)=O\left(\frac{1}{z^{2}}\right) \quad ; \quad \Omega^{1}(z)=O\left(\frac{1}{z^{2}}\right) \quad \text { for } z \rightarrow \infty .
$$

\subsection{Definition of some potentials associated to $\Phi^{1}(z)$ and $\Omega^{1}(z)$}

A major difficulty in the determination of the potentials $\Phi^{1}(z)$ and $\Omega^{1}(z)$ is that by equations (10) and (13), they diverge like $\left(z-z_{0}\right)^{-3 / 2}$ and therefore more quickly than 
$\left(z-z_{0}\right)^{-1}$ near each crack tip $z_{0}$. The problem is that if a function is analytic except at some point $z_{0}$ but behaves in this way near it, its singularity at this point is not removable.

To circumvent this difficulty, we shall look for some functions $\Phi_{A}^{1}(z), \Omega_{A}^{1}(z)$, known explicitly, "associated" or "adapted" to the potentials $\Phi^{1}(z), \Omega^{1}(z)$ (whence the index ${ }_{A}$ ) in the sense that are required to

(1) be defined and analytic on the same domain, that is over the whole complex plane except on the real intervals $[2 n d-c, 2 n d+c], n \in \mathbb{Z}$;

(2) be periodic of period $2 d$;

(3) behave in the same way near each crack tip in the sense that

$$
\left\{\begin{array}{l}
\Phi_{*}^{1}(z) \equiv\left(\Phi^{1}-\Phi_{A}^{1}\right)(z)=O\left[(z-2 n d \mp c)^{-1 / 2}\right] \\
\Omega_{*}^{1}(z) \equiv\left(\Omega^{1}-\Omega_{A}^{1}\right)(z)=O\left[(z-2 n d \mp c)^{-1 / 2}\right]
\end{array} \quad \text { for } z \rightarrow 2 n d \pm c ;\right.
$$

(4) behave also in the same way near infinity, that is vanish at least as quickly as $z^{-2}$ there.

Once such functions are found, we shall write the boundary conditions (27) on the less singular potentials $\Phi_{*}^{1}(z), \Omega_{*}^{1}(z)$ defined by equations $(29)$.

The idea is to look for the functions $\Phi_{A}^{1}(z), \Omega_{A}^{1}(z)$ in the form

$$
\left\{\begin{array}{l}
\Phi_{A}^{1}(z) \equiv \frac{f(z)}{X^{3}(z)}=\frac{f(z)}{\left[\sin ^{2}\left(\frac{\pi z}{2 d}\right)-\sin ^{2}\left(\frac{\pi c}{2 d}\right)\right]^{3 / 2}} \\
\Omega_{A}^{1}(z) \equiv \frac{g(z)}{X^{3}(z)}=\frac{g(z)}{\left[\sin ^{2}\left(\frac{\pi z}{2 d}\right)-\sin ^{2}\left(\frac{\pi c}{2 d}\right)\right]^{3 / 2}}
\end{array}\right.
$$

for some entire functions $f(z), g(z)$. Consider for instance the function $\Phi_{A}^{1}(z)$.

- Formula $(30)_{1}$ automatically ensures the satisfaction of condition (1) above.

- The functions $X(z)$ and $X^{3}(z)$ being antiperiodic of antiperiod $2 d$, condition (2) requires that $f(z)$ also be antiperiodic of antiperiod $2 d$.

- For such a function, it suffices to examine condition (3) near the sole points $\pm c$. Now an asymptotic study of the function $X^{3}(z)$ near these points reveals that

$$
\begin{cases}\Phi_{A}^{1}(z)=f(c)\left[\frac{\pi}{2 d} \sin \left(\frac{\pi c}{d}\right)\right]^{-3 / 2}(z-c)_{-}^{-3 / 2}+O\left[(z-c)^{-1 / 2}\right] & \text { for } z \rightarrow c \\ \Phi_{A}^{1}(z)=i f(-c)\left[\frac{\pi}{2 d} \sin \left(\frac{\pi c}{d}\right)\right]^{-3 / 2}(z+c)_{+}^{-3 / 2}+O\left[(z+c)^{-1 / 2}\right] & \text { for } z \rightarrow-c .\end{cases}
$$

Comparing with the asymptotic formulae $(10)_{1}$ and $(13)_{1}$ (with $a \equiv-c$ and $b \equiv c$ ), one concludes that condition (3) requires that

$$
f(c)=-f(-c)=\frac{i \pi c K^{0}}{16 d^{3 / 2}}\left[\sin \left(\frac{\pi c}{d}\right)\right]^{3 / 2} .
$$

Many entire functions, of course, are antiperiodic of antiperiod $2 d$ and take such values 
at the points $\pm c$; but the simplest of all is defined by

$$
f(z) \equiv \frac{i \pi c K^{0}}{16 d^{3 / 2}}\left[\sin \left(\frac{\pi c}{d}\right)\right]^{3 / 2} \frac{\sin \left(\frac{\pi z}{2 d}\right)}{\sin \left(\frac{\pi c}{2 d}\right)}
$$

- Finally with such a definition of $f(z)$, condition (4) is satisfied, $\Phi_{A}^{1}(z)$ vanishing at infinity more quickly than any power function of $z$.

A similar reasoning leads to the following formula for the function $g(z)$ :

$$
g(z) \equiv \frac{\pi c\left(i K_{I}^{0}-3 K_{I I}^{0}\right)}{16 d^{3 / 2}}\left[\sin \left(\frac{\pi c}{d}\right)\right]^{3 / 2} \frac{\sin \left(\frac{\pi z}{2 d}\right)}{\sin \left(\frac{\pi c}{2 d}\right)}
$$

In conclusion, the functions $\Phi_{A}^{1}(z), \Omega_{A}^{1}(z)$ selected are defined by

$$
\left\{\begin{array}{l}
\Phi_{A}^{1}(z) \equiv \frac{i \pi c K^{0}}{16 d^{3 / 2}} \frac{\left[\sin \left(\frac{\pi c}{d}\right)\right]^{3 / 2}}{\sin \left(\frac{\pi c}{2 d}\right)} \frac{\sin \left(\frac{\pi z}{2 d}\right)}{\left[\sin ^{2}\left(\frac{\pi z}{2 d}\right)-\sin ^{2}\left(\frac{\pi c}{2 d}\right)\right]^{3 / 2}} \\
\Omega_{A}^{1}(z) \equiv \frac{\pi c\left(i K_{I}^{0}-3 K_{I I}^{0}\right)}{16 d^{3 / 2}} \frac{\left[\sin \left(\frac{\pi c}{d}\right)\right]^{3 / 2}}{\sin \left(\frac{\pi c}{2 d}\right)} \frac{\sin 2}{\left[\sin ^{2}\left(\frac{\pi z}{2 d}\right)-\sin ^{2}\left(\frac{\pi c}{2 d}\right)\right]^{3 / 2}}
\end{array}\right.
$$

\subsection{Determination of Muskhelishvili's potentials $\Phi^{1}(z)$ and $\Omega^{1}(z)$}

Equations (27) read, in terms of the potentials $\Phi_{*}^{1}(z)$ and $\Omega_{*}^{1}(z)$ defined by equations (29):

$$
\left\{\begin{array}{l}
\left(\Phi_{*}^{1}+\Omega_{*}^{1}\right)\left(t^{+}\right)+\left(\Phi_{*}^{1}+\Omega_{*}^{1}\right)\left(t^{-}\right)=2 p(t) \\
\left(\Phi_{*}^{1}-\Omega_{*}^{1}\right)\left(t^{+}\right)-\left(\Phi_{*}^{1}-\Omega_{*}^{1}\right)\left(t^{-}\right)=2 q(t)
\end{array} \quad \text { for } t \in(2 n d-c, 2 n d+c), n \in \mathbb{Z}\right.
$$

where

$$
\left\{\begin{aligned}
p(t) \equiv & -2 i \frac{d}{d t}\left\{(t-2 n d) \operatorname{Re}\left[\Phi^{0}\left(t^{+}\right)+\Phi^{0}\left(t^{-}\right)\right]\right\} \\
& -\frac{1}{2}\left(\Phi_{A}^{1}+\Omega_{A}^{1}\right)\left(t^{+}\right)-\frac{1}{2}\left(\Phi_{A}^{1}+\Omega_{A}^{1}\right)\left(t^{-}\right) \\
q(t) \equiv & -2 i \frac{d}{d t}\left\{(t-2 n d) \operatorname{Re}\left[\Phi^{0}\left(t^{+}\right)-\Phi^{0}\left(t^{-}\right)\right]\right\} \\
& -\frac{1}{2}\left(\Phi_{A}^{1}-\Omega_{A}^{1}\right)\left(t^{+}\right)+\frac{1}{2}\left(\Phi_{A}^{1}-\Omega_{A}^{1}\right)\left(t^{-}\right)
\end{aligned} \text {for } t \in(2 n d-c, 2 n d+c), n \in \mathbb{Z} .\right.
$$

Equations $(23)_{1}$ and (33) permit to calculate the functions $p(t)$ and $q(t)$ explicitly; the calculation is simple for $p(t)$, less so for $q(t)$ but nevertheless straightforward. The results are as follows, considering only values of $t$ in the interval $(-c, c)$, which is possible since 
the functions are obviously periodic of period $2 d$ :

$$
\left\{\begin{aligned}
p(t)=i\left(\sigma_{22}^{\infty}-\sigma_{11}^{\infty}\right) & \\
q(t)=i \sigma_{12}^{\infty}\{ & \frac{\sin \left(\frac{\pi t}{2 d}\right)+\frac{\pi t}{2 d} \cos \left(\frac{\pi t}{2 d}\right)}{\left[\sin ^{2}\left(\frac{\pi c}{2 d}\right)-\sin ^{2}\left(\frac{\pi t}{2 d}\right)\right]^{1 / 2}} \\
& \left.+\frac{\frac{\pi t}{2 d} \sin \left(\frac{\pi t}{d}\right)-\frac{\pi c}{2 d} \sin \left(\frac{\pi c}{d}\right)}{\left[\sin ^{2}\left(\frac{\pi c}{2 d}\right)-\sin ^{2}\left(\frac{\pi t}{2 d}\right)\right]^{3 / 2}} \sin \left(\frac{\pi t}{2 d}\right)\right\}
\end{aligned}\right.
$$

Here the fact that $p(t)$ is a constant makes equation $(34)_{1}$ easier to solve. Indeed, by equation (18), it may be rewritten in the form

$$
\begin{aligned}
& \left(\Phi_{*}^{1}+\Omega_{*}^{1}\right)\left(t^{+}\right)+i\left(\sigma_{11}^{\infty}-\sigma_{22}^{\infty}\right)+\left(\Phi_{*}^{1}+\Omega_{*}^{1}\right)\left(t^{-}\right)+i\left(\sigma_{11}^{\infty}-\sigma_{22}^{\infty}\right)=0 \\
\Leftrightarrow & X\left(t^{+}\right)\left[\left(\Phi_{*}^{1}+\Omega_{*}^{1}\right)\left(t^{+}\right)+i\left(\sigma_{11}^{\infty}-\sigma_{22}^{\infty}\right)\right]-X\left(t^{-}\right)\left[\left(\Phi_{*}^{1}+\Omega_{*}^{1}\right)\left(t^{-}\right)+i\left(\sigma_{11}^{\infty}-\sigma_{22}^{\infty}\right)\right]=0
\end{aligned}
$$

for $t \in(2 n d-c, 2 n d+c), n \in \mathbb{Z}$.

Thus the function $X(z)\left[\left(\Phi_{*}^{1}+\Omega_{*}^{1}\right)(z)+i\left(\sigma_{11}^{\infty}-\sigma_{22}^{\infty}\right)\right]$, which is a priori analytic over the whole complex plane except on the intervals $[2 n d-c, 2 n d+c]$, is continuous across the intervals $(2 n d-c, 2 n d+c)$ and therefore analytic everywhere except perhaps at the points $2 n d \pm c$. Since, by equations (17) and (29), it remains finite at these points, it is in fact analytic over the whole complex plane. Hence

$$
\left(\Phi_{*}^{1}+\Omega_{*}^{1}\right)(z) \equiv \frac{Q(z)}{X(z)}+i\left(\sigma_{22}^{\infty}-\sigma_{11}^{\infty}\right) \quad \text { for all } z \in \mathbb{C}
$$

where $Q(z)$ is an entire function. Following then the same reasoning as in Subsection 4.1 for the function $\left(\Phi^{0}+\Omega^{0}\right)(z)$, one concludes from the finiteness of $\left(\Phi_{*}^{1}+\Omega_{*}^{1}\right)(z)$ at infinity, arising from equations $(28)$ and (33), that $Q(z)$ must be of the form

$$
Q(z) \equiv C_{1} \cos \left(\frac{\pi z}{2 d}\right)+D_{1} \sin \left(\frac{\pi z}{2 d}\right)
$$

for some coefficients $C_{1}, D_{1}$. Then, by equation (19),

$$
\left(\Phi_{*}^{1}+\Omega_{*}^{1}\right)\left(i x_{2}\right)=\frac{Q\left(i x_{2}\right)}{X\left(i x_{2}\right)}+i\left(\sigma_{22}^{\infty}-\sigma_{11}^{\infty}\right) \rightarrow \mp i C_{1}+D_{1}+i\left(\sigma_{22}^{\infty}-\sigma_{11}^{\infty}\right) \quad \text { for } x_{2} \rightarrow \pm \infty .
$$

But both of these limits must be zero by equations (28) and (33), so that the values of $C_{1}$ and $D_{1}$ are

$$
C_{1}=0 \quad ; \quad D_{1}=i\left(\sigma_{11}^{\infty}-\sigma_{22}^{\infty}\right),
$$

and it follows that

$$
\left(\Phi_{*}^{1}+\Omega_{*}^{1}\right)(z)=i\left(\sigma_{11}^{\infty}-\sigma_{22}^{\infty}\right)\left\{\frac{\sin \left(\frac{\pi z}{2 d}\right)}{\left[\sin ^{2}\left(\frac{\pi z}{2 d}\right)-\sin ^{2}\left(\frac{\pi c}{2 d}\right)\right]^{1 / 2}}-1\right\} \quad \text { for all } z \in \mathbb{C} .
$$


In order to now determine the function $\left(\Phi_{*}^{1}-\Omega_{*}^{1}\right)(z)$, introduce the auxiliary function ${ }^{4}$

$$
\begin{aligned}
\chi(z) & \equiv \frac{1}{i \pi} \sum_{n=-\infty}^{+\infty} \int_{2 n d-c}^{2 n d+c} \frac{q(t) d t}{t-z} \\
& =\frac{1}{i \pi} \int_{-c}^{c}\left(\sum_{n=-\infty}^{+\infty} \frac{1}{t+2 n d-z}\right) q(t) d t=\frac{i}{2 d} \int_{-c}^{c} \cot \left[\frac{\pi(z-t)}{2 d}\right] q(t) d t
\end{aligned}
$$

where use has been made of the periodicity of $q(t)$ and the series representation of the cotangent, $\sum_{n=-\infty}^{+\infty} \frac{1}{x+n}=\pi \cot (\pi x)$ (Gradshteyn and Ryzhik (1980), formula 1.421.3). By Plemelj's formula (Muskhelishvili (1953), p. 263),

$$
\chi\left(t^{+}\right)-\chi\left(t^{-}\right)=2 q(t) \quad \text { for } t \in(2 n d-c, 2 n d+c), n \in \mathbb{Z}
$$

so that equation $(34)_{2}$ may be rewritten as

$$
\left(\Phi_{*}^{1}-\Omega_{*}^{1}-\chi\right)\left(t^{+}\right)-\left(\Phi_{*}^{1}-\Omega_{*}^{1}-\chi\right)\left(t^{-}\right)=0 \quad \text { for } t \in(2 n d-c, 2 n d+c), n \in \mathbb{Z} .
$$

Reasoning like for the function $X(z)\left[\left(\Phi_{*}^{1}+\Omega_{*}^{1}\right)(z)+i\left(\sigma_{11}^{\infty}-\sigma_{22}^{\infty}\right)\right]$, one concludes that the function $\left(\Phi_{*}^{1}-\Omega_{*}^{1}-\chi\right)(z)$, being continuous across the intervals $(2 n d-c, 2 n d+c)$, is analytic on the whole complex plane except perhaps at the points $2 n d \pm c$; but its singularities at these points are removable by equations (29) and the very definition (39) of $\chi(z)^{5}$, so that it is an entire function. Since it is zero at infinity by equations (28), (33) and (39), by Liouville's theorem, it is zero everywhere. Hence

$$
\left(\Phi_{*}^{1}-\Omega_{*}^{1}\right)(z)=\chi(z)=\frac{i}{2 d} \int_{-c}^{c} \cot \left[\frac{\pi(z-t)}{2 d}\right] q(t) d t \quad \text { for all } z \in \mathbb{C} .
$$

Combination of the definitions (29) of $\Phi_{*}^{1}(z), \Omega_{*}^{1}(z)$, equations (38) and (40) yields the final expressions of $\Phi^{1}(z)$ and $\Omega^{1}(z)$ :

$$
\left\{\begin{aligned}
\Phi^{1}(z)= & \Phi_{A}^{1}(z)+\Phi_{*}^{1}(z), \\
\Phi_{*}^{1}(z)= & \frac{i}{4 d} \int_{-c}^{c} \cot \left[\frac{\pi(z-t)}{2 d}\right] q(t) d t \\
& +\frac{i}{2}\left(\sigma_{11}^{\infty}-\sigma_{22}^{\infty}\right)\left\{\frac{\sin \left(\frac{\pi z}{2 d}\right)}{\left[\sin ^{2}\left(\frac{\pi z}{2 d}\right)-\sin ^{2}\left(\frac{\pi c}{2 d}\right)\right]^{1 / 2}}-1\right\} \quad \text { for all } z \in \mathbb{C} \\
\Omega^{1}(z)= & \Omega_{A}^{1}(z)+\Omega_{*}^{1}(z), \\
\Omega_{*}^{1}(z)= & -\frac{i}{4 d} \int_{-c}^{c} \cot \left[\frac{\pi(z-t)}{2 d}\right] q(t) d t \\
& +\frac{i}{2}\left(\sigma_{11}^{\infty}-\sigma_{22}^{\infty}\right)\left\{\frac{\sin \left(\frac{\pi z}{2 d}\right)}{\left[\sin ^{2}\left(\frac{\pi z}{2 d}\right)-\sin ^{2}\left(\frac{\pi c}{2 d}\right)\right]^{1 / 2}}-1\right\}
\end{aligned}\right.
$$

$\overline{4}$ The infinite series $\sum_{n=-\infty}^{+\infty}(\ldots)$ appearing here and just below must be understood in the sense $\lim _{N \rightarrow+\infty} \sum_{n=-N}^{N}(\ldots)$.

5 This equation implies that $\chi(z)$ diverges like $(z-2 n d \mp c)^{-1 / 2}$ near the points $2 n d \pm c$; see Subsection 5.4 below. 
where $\Phi_{A}^{1}(z), \Omega_{A}^{1}(z)$ and $q(t)$ are given by equations (33) and $(36)_{2}$.

\subsection{Expressions of the stress intensity factors}

Again, the SIF being identical at all crack tips for obvious symmetry reasons, their firstorder expansions in powers of $\alpha$ will simply be denoted $K_{I}^{0}+\alpha K_{I}^{1}+O\left(\alpha^{2}\right), K_{I I}^{0}+\alpha K_{I I}^{1}+$ $O\left(\alpha^{2}\right)$, without a lower index ${ }_{a}$ or ${ }_{b}$ like in Subsection 3.2. The expressions of $K_{I}^{0}$ and $K_{I I}^{0}$ are given by equations $(24)_{1,2}$ and we are looking here for those of $K_{I}^{1}$ and $K_{I I}^{1}$. This will be done by examining the asymptotic expression of the function $\Phi_{*}^{1}(z)$ near the tip $c$.

By equation $(29)_{1}$, this expression must be of the form

$$
\Phi_{*}^{1}(z)=\frac{K_{*}^{1}}{2[2 \pi(z-c)]_{-}^{1 / 2}}+O(1) \quad \text { for } z \rightarrow c
$$

for some coefficient $K_{*}^{1}$. The first task is to calculate this coefficient using the expression $(41)_{2}$ of $\Phi_{*}^{1}(z)$. This will be done by considering points of the form $z=c+r, r>0, r \rightarrow 0$, and writing $K_{*}^{1}$ in the form

$$
K_{*}^{1}=\lim _{r>0, r \rightarrow 0} 2 \sqrt{2 \pi r} \Phi_{*}^{1}(z=c+r) .
$$

By equation $(41)_{2}$, this limit consists of two contributions arising from the integral term and the term $\frac{i}{2}\left(\sigma_{11}^{\infty}-\sigma_{22}^{\infty}\right)\{\ldots\}$, respectively. The first is connected to the limit

$$
L \equiv \lim _{r>0, r \rightarrow 0} \int_{-c}^{c} \sqrt{r} \cot \left[\frac{\pi(c+r-t)}{2 d}\right] q(t) d t
$$

the calculation of this limit is presented in Appendix A, and the result is

$$
L=2 i \sigma_{12}^{\infty} \sqrt{\frac{d^{3}}{\pi} \tan \left(\frac{\pi c}{2 d}\right)}\left[1+\frac{\pi c / d}{\sin (\pi c / d)}\right] .
$$

The calculation of the second contribution is elementary. Adding the two contributions, one finally finds that

$$
K_{*}^{1}=\sqrt{2 d \tan \left(\frac{\pi c}{2 d}\right)}\left\{i\left(\sigma_{11}^{\infty}-\sigma_{22}^{\infty}\right)-\sigma_{12}^{\infty}\left[1+\frac{\pi c / d}{\sin (\pi c / d)}\right]\right\} .
$$

We shall now obtain the asymptotic expression of the function $\Phi_{*}^{1}(z)$ near the tip $c$ in another way, by using those of the functions $\Phi^{1}(z)$ and $\Phi_{A}^{1}(z)$. First, equations $(10)_{1}$ (with $a \equiv-c$ and $b \equiv c$ ) and $(29)_{1}$ imply that the expansion of $\Phi_{A}^{1}(z)$ near the tip $c$ must read

$$
\Phi_{A}^{1}(z)=\frac{i c K^{0}}{4 \sqrt{2 \pi}(z-c)_{-}^{3 / 2}}+\frac{K_{A}^{1}}{2[2 \pi(z-c)]_{-}^{1 / 2}}+O(1) \quad \text { for } z \rightarrow c
$$


for some constant $K_{A}^{1}$. To calculate $K_{A}^{1}$, it suffices to expand the expression $(33)_{1}$ of $\Phi_{A}^{1}(z)$ at the point $z=c+r$ up to order $r^{-1 / 2}$ and to identify the coefficient of the term of this order to $\frac{K_{A}^{1}}{2 \sqrt{2 \pi}}$; one thus gets after some calculation

$$
K_{A}^{1}=\left(i \sigma_{22}^{\infty}+\sigma_{12}^{\infty}\right) \sqrt{2 d \tan \left(\frac{\pi c}{2 d}\right)}\left[\frac{\pi c}{4 d} \cot \left(\frac{\pi c}{2 d}\right)-\frac{3 \pi c}{8 d} \cot \left(\frac{\pi c}{d}\right)\right]
$$

Second, subtracting equation (47) from equation (10) ${ }_{1}$ (with $a \equiv-c$ and $b \equiv c$ ), one gets

$$
\Phi_{*}^{1}(z)=\Phi^{1}(z)-\Phi_{A}^{1}(z)=\frac{K^{1}+i K^{0} / 2-i c B^{0}-K_{A}^{1}}{2[2 \pi(z-c)]_{-}^{1 / 2}}+O(1) \quad \text { for } z \rightarrow c
$$

Comparing equations (42) and (49), one concludes that

$$
K^{1}=K_{A}^{1}+K_{*}^{1}-\frac{i K^{0}}{2}+i c B^{0}
$$

Inserting the expressions $(24)_{1,2,4}$ of $K_{I}^{0}, K_{I I}^{0}, B^{0}$, (46) of $K_{*}^{1}$ and (48) of $K_{A}^{1}$ into this equation and identifying the real and imaginary parts of $K^{1} \equiv K_{I}^{1}-i K_{I I}^{1}$, one finally gets

$$
\left\{\begin{array}{l}
K_{I}^{1}=-\frac{\sigma_{12}^{\infty}}{2} \sqrt{2 d \tan \left(\frac{\pi c}{2 d}\right)}\left[3+\frac{\pi c / d}{\sin (\pi c / d)}\right] \\
K_{I I}^{1}=\sqrt{2 d \tan \left(\frac{\pi c}{2 d}\right)}\left\{-\sigma_{11}^{\infty}+\frac{\sigma_{22}^{\infty}}{2}\left[3-\frac{\pi c / d}{\sin (\pi c / d)}\right]\right\}
\end{array}\right.
$$

Note the remarkable simplicity of these formulae, in complete contrast with the lengthy and elaborate calculations required to obtain them.

\section{A "composite" approximation}

Equations (24) $)_{1,2}$ and (51) provide the following expressions of the SIF, applicable to small values of the tilt angle $\alpha$ but arbitrary values of the ratio $c / d$ :

$$
\left\{\begin{array}{l}
K_{I}=\sqrt{2 d \tan \left(\frac{\pi c}{2 d}\right)}\left\{\sigma_{22}^{\infty}-\alpha \frac{\sigma_{12}^{\infty}}{2}\left[3+\frac{\pi c / d}{\sin (\pi c / d)}\right]+O\left(\alpha^{2}\right)\right\} \\
K_{I I}=\sqrt{2 d \tan \left(\frac{\pi c}{2 d}\right)}\left\{\sigma_{12}^{\infty}-\alpha \sigma_{11}^{\infty}+\alpha \frac{\sigma_{22}^{\infty}}{2}\left[3-\frac{\pi c / d}{\sin (\pi c / d)}\right]+O\left(\alpha^{2}\right)\right\} .
\end{array}\right.
$$

However, there is another case where the SIF may be calculated trivially, now for arbitrary values of the tilt angle: namely that of a vanishingly small ratio $c / d$. Indeed in such a case the cracks become independent of each other so that the SIF become identical to those for a unique crack inclined over the horizontal axis $O x_{1}$, which are easily obtained by 
expressing the stress tensor at infinity in the frame "adapted" to the crack:

$$
\left\{\begin{array}{l}
K_{I} \sim \sqrt{\frac{\pi c}{\cos \alpha}}\left[\sigma_{11}^{\infty} \frac{1-\cos (2 \alpha)}{2}+\sigma_{22}^{\infty} \frac{1+\cos (2 \alpha)}{2}-\sigma_{12}^{\infty} \sin (2 \alpha)\right] \\
K_{I I} \sim \sqrt{\frac{\pi c}{\cos \alpha}}\left[-\sigma_{11}^{\infty} \frac{\sin (2 \alpha)}{2}+\sigma_{22}^{\infty} \frac{\sin (2 \alpha)}{2}+\sigma_{12}^{\infty} \cos (2 \alpha)\right]
\end{array} \quad \text { for } c / d \rightarrow 0\right.
$$

(The factor $1 / \sqrt{\cos \alpha}$ in these expressions arises from the fact that $2 c$ has been defined in Section 2 as the projected length of the crack onto the axis $O x_{1}$; hence its true length is $2 c / \cos \alpha$ ). One may take advantage of expressions (53) to propose the following approximate extension of equations (52) to larger tilt angles:

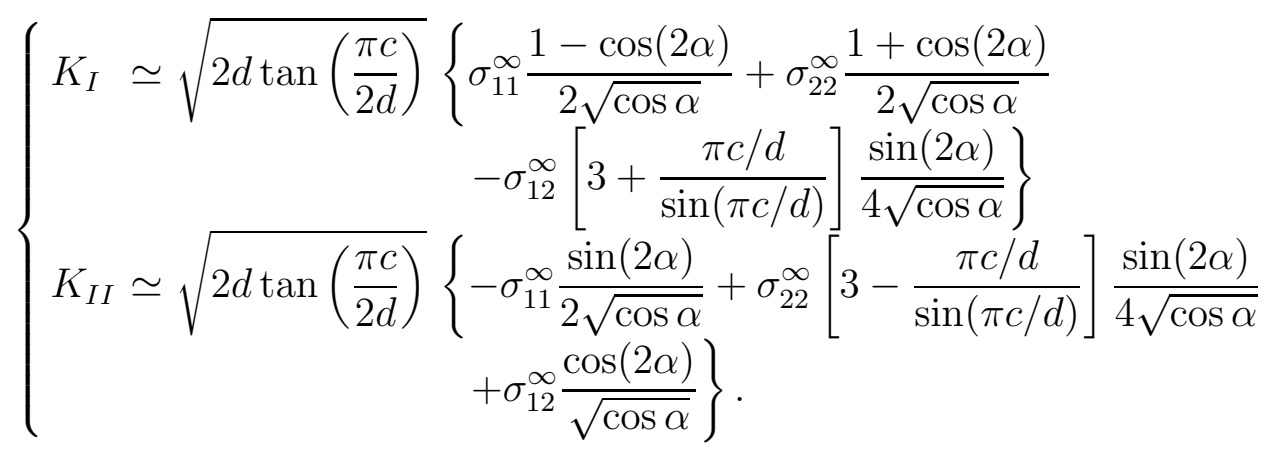

The "composite" approximations (54) reduce to expressions (52) at order 1 in $\alpha$ for all values of $c / d$, but also match expressions (53) for all values of $\alpha$ in the limit $c / d \rightarrow 0$. Hence they may be expected to provide a better representation of the SIF than equations (52).

\section{$7 \quad$ Finite element computations}

Numerical computations of the SIF for the geometrical configuration considered have been performed by Fleck (1991), Wang and Feng (2001) and Chen et al. (2009), but for a few values of the pair $(c / d, \alpha)$ only. More systematic calculations have been deemed necessary and performed using the CAST3M finite element code developed by the French Commissariat à l'Energie Atomique.

Thanks to periodicity, the calculations are limited to a rectangular region $-d \leq x_{1} \leq d$, $-H \leq x_{2} \leq H$ of large vertical dimension $2 H$. They are performed in plane strain, imposing (i) conditions of homogeneous boundary strain on the upper and lower sides:

$$
\mathbf{u}(\mathbf{x})=\boldsymbol{\epsilon}^{\infty} \cdot \mathbf{x} \text { for } x_{2}= \pm H
$$

where $\mathbf{u}(\mathbf{x})$ denotes the displacement and $\boldsymbol{\epsilon}^{\infty}$ the remote strain tensor, and (ii) conditions of periodicity on the lateral sides:

$$
\mathbf{u}(\mathbf{x})-\mathbf{u}\left(\mathbf{x}-2 d \mathbf{e}_{1}\right)=\epsilon^{\infty} .2 d \mathbf{e}_{1} \quad \text { for } x_{1}=d .
$$

In order to compare the SIF determined numerically to those predicted by equations (54), 
we write them in the form

$$
\left\{\begin{array}{l}
K_{I} \equiv \sqrt{2 d \tan \left(\frac{\pi c}{2 d}\right)}\left[F_{11}^{I}\left(\frac{c}{d}, \alpha\right) \sigma_{11}^{\infty}+F_{22}^{I}\left(\frac{c}{d}, \alpha\right) \sigma_{22}^{\infty}+F_{12}^{I}\left(\frac{c}{d}, \alpha\right) \sigma_{12}^{\infty}\right] \\
K_{I I} \equiv \sqrt{2 d \tan \left(\frac{\pi c}{2 d}\right)}\left[F_{11}^{I I}\left(\frac{c}{d}, \alpha\right) \sigma_{11}^{\infty}+F_{22}^{I I}\left(\frac{c}{d}, \alpha\right) \sigma_{22}^{\infty}+F_{12}^{I I}\left(\frac{c}{d}, \alpha\right) \sigma_{12}^{\infty}\right]
\end{array}\right.
$$

where the factor $\sqrt{2 d \tan \left(\frac{\pi c}{2 d}\right)}$ is introduced conventionally in reference to the solution for a zero tilt angle, see equations $(24)_{1,2}$ above. The expressions of the functions $F_{i j}^{p}(c / d, \alpha)$ resulting from the composite approximation proposed (54) are as follows:

$$
\left\{\begin{array}{l}
F_{11}^{I}\left(\frac{c}{d}, \alpha\right) \simeq \frac{1-\cos (2 \alpha)}{2 \sqrt{\cos \alpha}} ; \quad F_{22}^{I}\left(\frac{c}{d}, \alpha\right) \simeq \frac{1+\cos (2 \alpha)}{2 \sqrt{\cos \alpha}} ; \\
F_{12}^{I}\left(\frac{c}{d}, \alpha\right) \simeq-\left[3+\frac{\pi c / d}{\sin (\pi c / d)}\right] \frac{\sin (2 \alpha)}{4 \sqrt{\cos \alpha}} ; \\
F_{11}^{I I}\left(\frac{c}{d}, \alpha\right) \simeq-\frac{\sin (2 \alpha)}{2 \sqrt{\cos \alpha}} ; \quad F_{22}^{I I}\left(\frac{c}{d}, \alpha\right) \simeq\left[3-\frac{\pi c / d}{\sin (\pi c / d)}\right] \frac{\sin (2 \alpha)}{4 \sqrt{\cos \alpha}} ; \\
F_{12}^{I I}\left(\frac{c}{d}, \alpha\right) \simeq \frac{\cos (2 \alpha)}{\sqrt{\cos \alpha}} .
\end{array}\right.
$$

To calculate these functions numerically, the values of the components of the tensor $\boldsymbol{\epsilon}^{\infty}$ are adjusted so as to get three distinct values of the triplet $\left(\sigma_{11}^{\infty}, \sigma_{22}^{\infty}, \sigma_{12}^{\infty}\right):(1,0,0),(0,1,0)$ and $(0,0,1)$, thus permitting to determine the functions $F_{11}^{p}(c / d, \alpha), F_{22}^{p}(c / d, \alpha)$ and $F_{12}^{p}(c / d, \alpha)$ separately.

The SIF at the tip $c$ are evaluated using Destuynder et al. (1983)'s " $G-\theta$ method", the accuracy of which is well established.

Most calculations have been performed for values of $c / d$ ranging from 0.1 to 0.5 and values of $\alpha$ ranging from $0^{\circ}$ to $45^{\circ}$. Figures 4 to 9 show the results obtained and compare them to those predicted by equations (58). These formulae may be observed to make a good job of reproducing the numerical results up to angles of at least $30^{\circ}-35^{\circ}$.

Some calculations have also been performed to assess the validity of expressions $(58)_{3,5}$ of the functions $F_{12}^{I}(c / d, \alpha)$ and $F_{22}^{I I}(c / d, \alpha)$ for larger values of the ratio $c / d$, up to 0.8 . These calculations consider only small values of $\alpha, 2.5^{\circ}$ and $5^{\circ}$, since Figures 6 and 8 make it clear that formulae (58) cannot be hoped to remain accurate when $c / d$ and $\alpha$ are both large. Figures 10 and 11 show the results obtained. One observes that formulae $(58)_{3,5}$ are still acceptable for large values of $c / d$, provided that $\alpha$ remains small.

\section{Discussion: tentative prediction of the stationary tilt angle of facets}

Formulae (58), validated by the finite element calculations just presented, open the way to theoretical predictions of the stationary tilt angles of well-developed facets no longer "feeling" the influence of the initial crack. This possibility is illustrated here in conclusion 


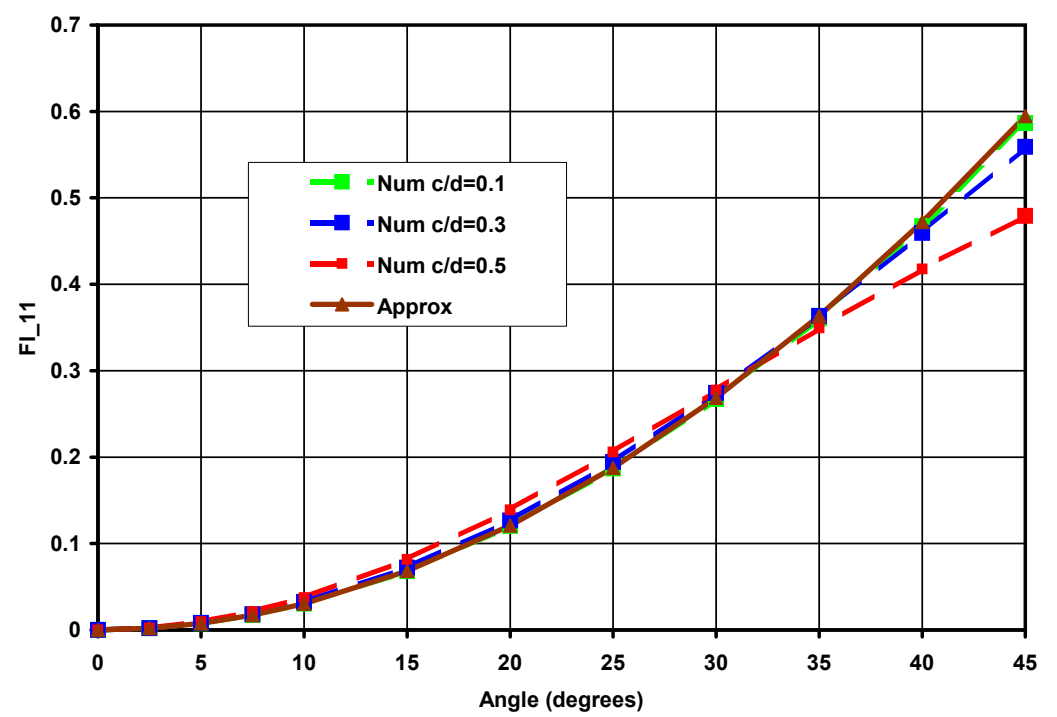

Fig. 4. The function $F_{11}^{I}(c / d, \alpha)$

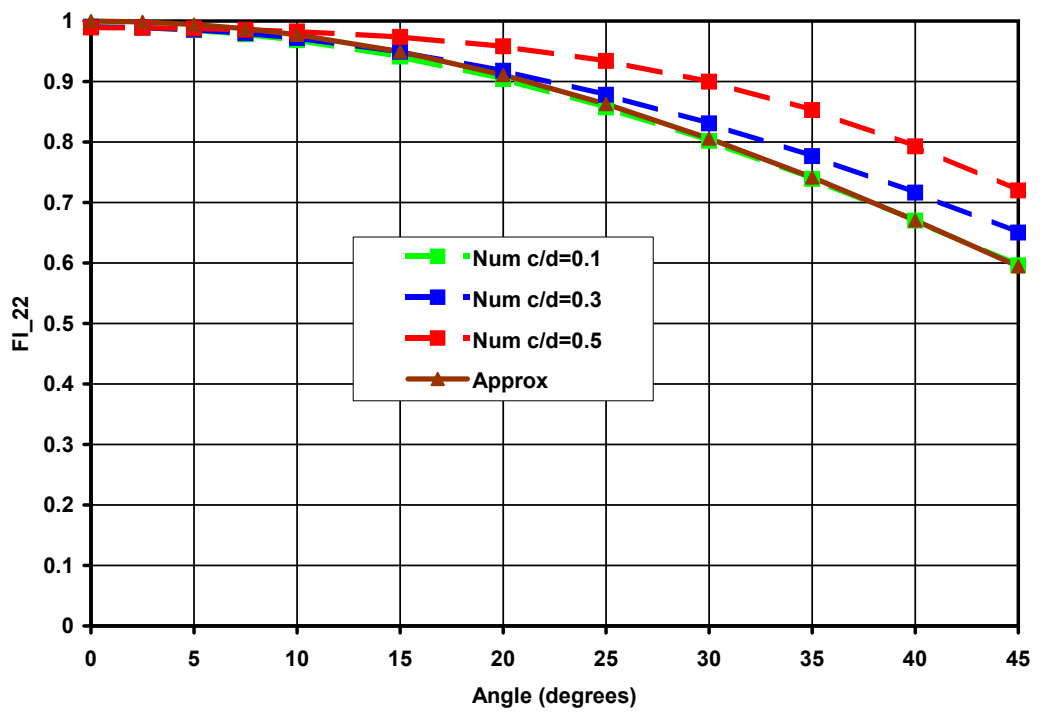

Fig. 5. The function $F_{22}^{I}(c / d, \alpha)$

by combining these formulae with a simple heuristic criterion for lateral propagation of the facets to get such a prediction.

It must be stressed that the prediction proposed is only tentative and certainly does not represent a final answer to the problem. More sophisticated proposals will be presented in 


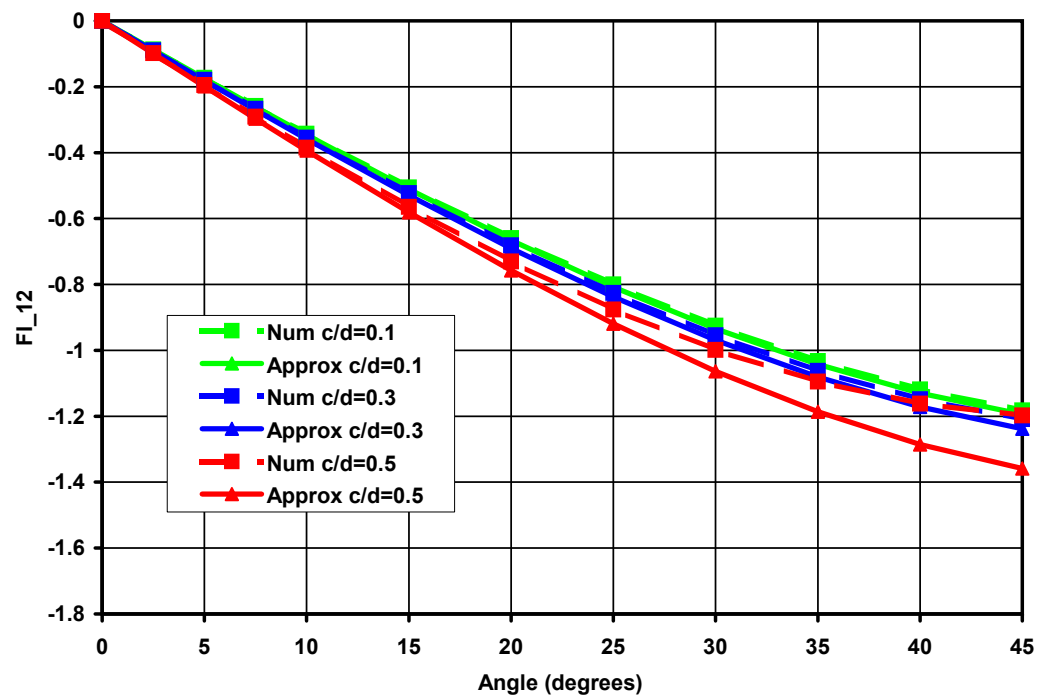

Fig. 6. The function $F_{12}^{I}(c / d, \alpha)$

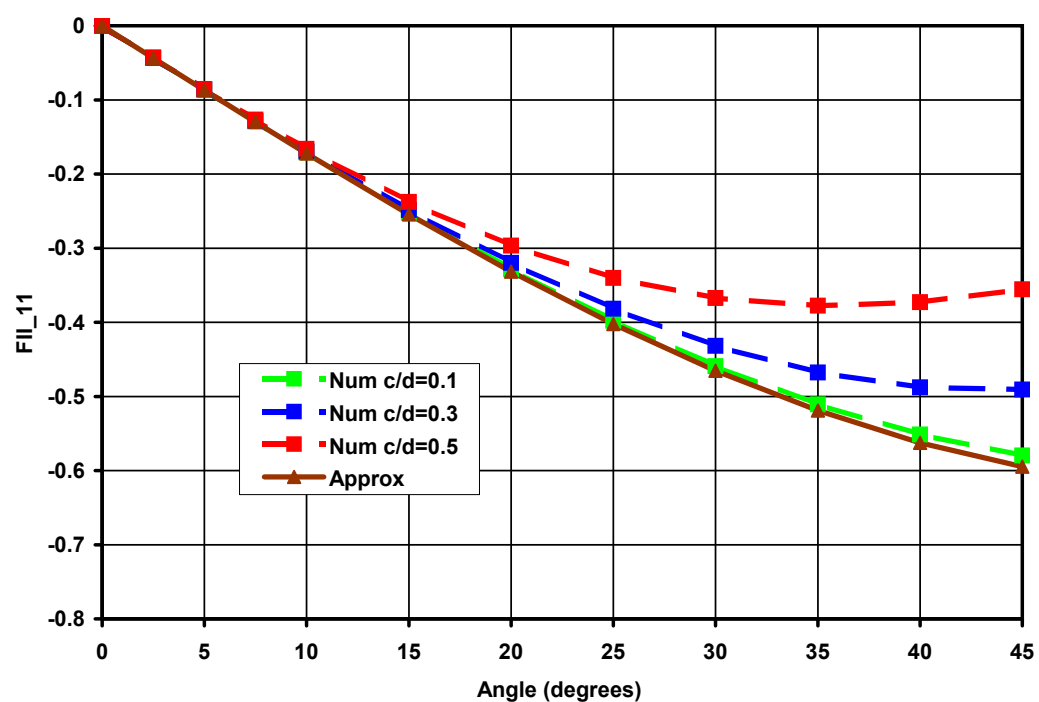

Fig. 7. The function $F_{11}^{I I}(c / d, \alpha)$

a future paper, together with comparisons with tilt angles observed in both experiments and numerical simulations using Karma et al. (2001)'s phase field model. 


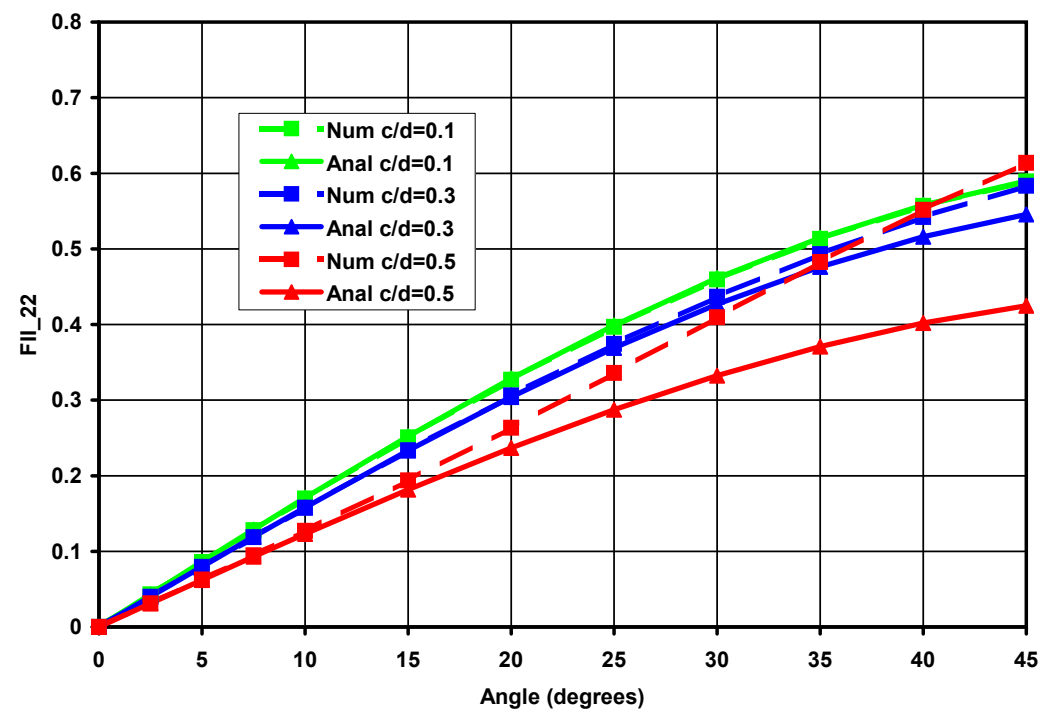

Fig. 8. The function $F_{22}^{I I}(c / d, \alpha)$

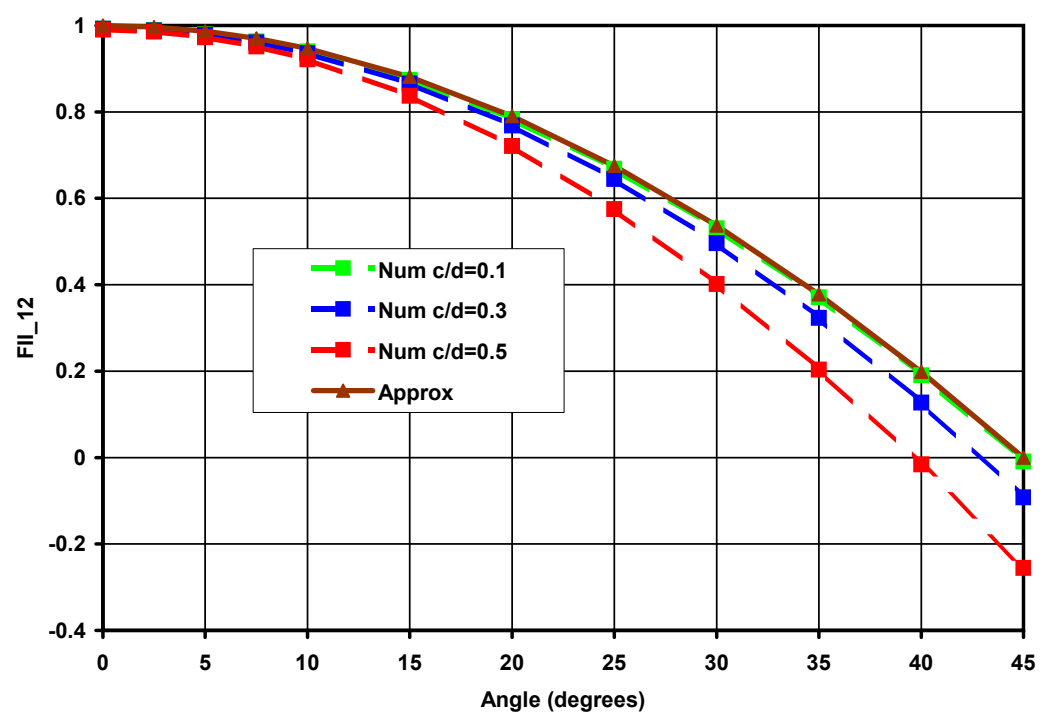

Fig. 9. The function $F_{12}^{I I}(c / d, \alpha)$

8.1 Expression of the stationary tilt angle in terms of the remote $2 D$ stresses

The heuristic postulate made is that the 2D SIF $K_{I I}$ is zero along the lateral sides of the facets. This hypothesis is just Goldstein and Salganik (1974)'s principle of local symmetry, 


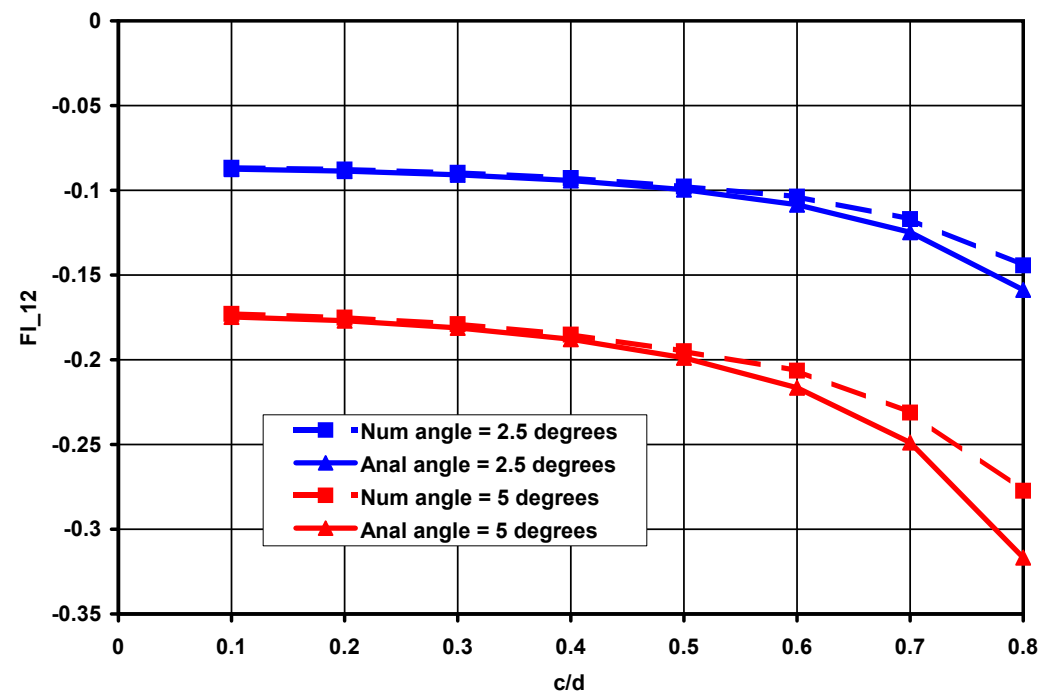

Fig. 10. The function $F_{12}^{I}(c / d, \alpha)$ for small tilt angles

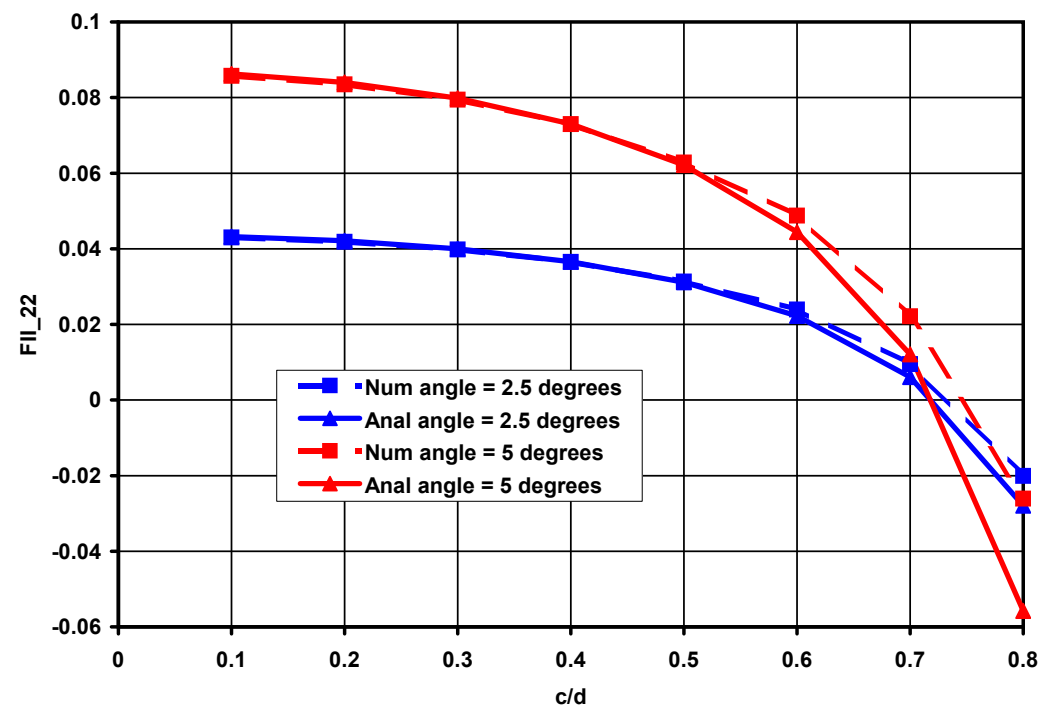

Fig. 11. The function $F_{22}^{I I}(c / d, \alpha)$ for small tilt angles

applied to lateral propagation of the facets: if $K_{I I}$ were not zero, lateral propagation of these facets would induce them to deviate out of their plane so that the tilt angle would not be stationary. 
Using the expression $(57)_{2}$ of $K_{I I}$ and dividing by $\sigma_{22}^{\infty}$, we thus get the condition

$$
\frac{\sigma_{11}^{\infty}}{\sigma_{22}^{\infty}} F_{11}^{I I}\left(\frac{c}{d}, \alpha\right)+F_{22}^{I I}\left(\frac{c}{d}, \alpha\right)+\frac{\sigma_{12}^{\infty}}{\sigma_{22}^{\infty}} F_{12}^{I I}\left(\frac{c}{d}, \alpha\right)=0
$$

which defines the tilt angle $\alpha$ as a function of the ratios $c / d, \sigma_{11}^{\infty} / \sigma_{22}^{\infty}$ and $\sigma_{12}^{\infty} / \sigma_{22}^{\infty}$. Condition (59), with the approximate expressions (58) $)_{4,5,6}$ of the functions $F_{i j}^{I I}(c / d, \alpha)$, is an algebraic equation of the second degree on $\tan \alpha$, the solution of which is elementary.

Since the tilt angle predicted by equation (59) depends upon the ratio $c / d$, it must vary in time as the width $2 c$ of the facets increases, in contradiction with its supposedly "stationary" character. Thus the prediction can only make sense for facets of moderate relative width, say $c / d \lesssim 0.5$, for which the functions $F_{i j}^{I I}(c / d, \alpha)$ depend only modestly on $c / d$. (In practice, facets of larger relative width are observed frequently, but not systematically; Goldstein and Osipenko (2012)'s experiments on gypsum and cheese, for instance, did involve ratios $c / d$ of the order of 0.5$)$.

\subsection{Expression of the stationary tilt angle in terms of the initial $3 D$ stress intensity factors}

In order to re-express equation (59) in terms of the ratio $K_{I I I}^{3 D} / K_{I}^{3 D}$, one must (i) calculate both the $3 \mathrm{D} \operatorname{SIF} K_{I}^{3 D}, K_{I I I}^{3 D}$ of the initial crack and the remote $2 \mathrm{D}$ stresses $\sigma_{11}^{\infty}, \sigma_{22}^{\infty}, \sigma_{12}^{\infty}$ (disregarding the influence of the initial crack) in terms of the load parameters, and (ii) eliminate these parameters so as to express $\sigma_{11}^{\infty} / \sigma_{22}^{\infty}$ and $\sigma_{12}^{\infty} / \sigma_{22}^{\infty}$ in terms of $K_{I I I}^{3 D} / K_{I}^{3 D}$. The expressions found will inevitably depend upon the type of loading envisaged, and so will the equation connecting the value of $\alpha$ to that of $K_{I I I}^{3 D} / K_{I}^{3 D}$. We shall consider three typical examples.

- Case 1: the body considered is infinite, contains an initial tunnel-crack lying in the plane $O x_{1} x_{3}$ of width $2 A$ in the direction $x_{3}$, and is subjected to uniform remote stresses $\sigma_{22}^{\infty}$, $\sigma_{12}^{\infty}$. Then $\sigma_{11}^{\infty} / \sigma_{22}^{\infty}=0$ and $K_{I}^{3 D}=\sigma_{22}^{\infty} \sqrt{\pi A}, K_{I I I}^{3 D}=-\sigma_{12}^{\infty} \sqrt{\pi A},{ }^{6}$ so that $\sigma_{12}^{\infty} / \sigma_{22}^{\infty}=$ $-K_{I I I}^{3 D} / K_{I}^{3 D}$. Equation (59) then becomes

$$
F_{22}^{I I}\left(\frac{c}{d}, \alpha\right)-\frac{K_{I I I}^{3 D}}{K_{I}^{3 D}} F_{12}^{I I}\left(\frac{c}{d}, \alpha\right)=0
$$

- Case 2: the body is a plate of infinite dimensions in the directions $x_{1}$ and $x_{3}$, large thickness $2 H$ in the direction $x_{2}$, and contains a semi-infinite initial crack; its upper and lower surfaces are subjected to the following boundary conditions:

$$
u_{1}(\mathbf{x})= \pm U_{1} \quad ; \quad u_{2}(\mathbf{x})= \pm U_{2} \quad ; \quad u_{3}(\mathbf{x})=0 \quad \text { for } x_{2}= \pm H
$$

Then $K_{I}^{3 D}=\frac{E}{(1+\nu) \sqrt{1-2 \nu}} \frac{U_{2}}{\sqrt{H}}$ and $K_{I I I}^{3 D}=-\frac{E}{1+\nu} \frac{U_{1}}{\sqrt{2 H}}$ where $E$ and $\nu$ denote Young's modulus and Poisson's ratio (Tada et al. (2000), pp. 268 and 270). Furthermore elementary calculations show that $\frac{\sigma_{11}^{\infty}}{\sigma_{22}^{\infty}}=\frac{\nu}{1-\nu}$ and $\frac{\sigma_{12}^{\infty}}{\sigma_{22}^{\infty}}=\frac{1-2 \nu}{2(1-\nu)} \frac{U_{1}}{U_{2}}=-\frac{1}{1-\nu} \sqrt{\frac{1-2 \nu}{2}} \frac{K_{I I I}^{3 D}}{K_{I}^{3 D}}$ by

$\overline{6}$ The minus sign arises from the definition of the axes $\mathrm{Ox}_{1}, \mathrm{Ox}_{2}, \mathrm{Ox}_{3}$, see Section 2. 
what precedes. Therefore equation (59) becomes

$$
\frac{\nu}{1-\nu} F_{11}^{I I}\left(\frac{c}{d}, \alpha\right)+F_{22}^{I I}\left(\frac{c}{d}, \alpha\right)-\frac{1}{1-\nu} \sqrt{\frac{1-2 \nu}{2}} \frac{K_{I I I}^{3 D}}{K_{I}^{3 D}} F_{12}^{I I}\left(\frac{c}{d}, \alpha\right)=0
$$

- Case 3: same as Case 2 except that the upper and lower surfaces are subjected to the conditions

$$
u_{1}(\mathbf{x})= \pm U_{1} \quad ; \quad u_{2}(\mathbf{x})= \pm U_{2} \quad ; \quad \sigma_{23}(\mathbf{x})=0 \quad \text { for } x_{2}= \pm H
$$

meaning that they are free to move in the direction $x_{3}$. Then the value of $K_{I I I}^{3 D}$ is unchanged and that of $K_{I}^{3 D}$ becomes $\frac{E}{1-\nu^{2}} \frac{U_{2}}{\sqrt{H}}$ (Tada et al. (2000), p. 269). Also, $\frac{\sigma_{11}^{\infty}}{\sigma_{22}^{\infty}}=\nu$ and $\frac{\sigma_{12}^{\infty}}{\sigma_{22}^{\infty}}=\frac{1-\nu}{2} \frac{U_{1}}{U_{2}}=-\frac{1}{\sqrt{2}} \frac{K_{I I I}^{3 D}}{K_{I}^{3 D}}$ by what precedes. Therefore equation (59) becomes

$$
\nu F_{11}^{I I}\left(\frac{c}{d}, \alpha\right)+F_{22}^{I I}\left(\frac{c}{d}, \alpha\right)-\frac{1}{\sqrt{2}} \frac{K_{I I I}^{3 D}}{K_{I}^{3 D}} F_{12}^{I I}\left(\frac{c}{d}, \alpha\right)=0
$$

Figure 12 shows, for the typical values $\nu=0.25$ and $c / d=0.5$, and using formulae $(58)_{4,5,6}$ for the functions $F_{i j}^{I I}(c / d, \alpha)$, the tilt angle predicted by equations (60), (62) and (64), as a function of the ratio $K_{I I I}^{3 D} / K_{I}^{3 D}$. The influence of the type of loading is moderate but evident here.

Figure 12 also displays the predictions of Pollard et al. (1982)'s equation (1). One observes that in all cases investigated the present estimate of the stationary tilt angle of facets is slightly lower than that of Pollard et al. (1982) of their initial tilt angle. Without anticipating too much on future detailed comparisons with tilt angles observed experimpentally, one may note that our predictions are bound to be somewhat better than those of Pollard et al. (1982) since, as mentioned in the Introduction, Pollard et al. (1982)'s ones were precisely noted by Lazarus et al. (2001b) to be somewhat too high.

\section{Final remark}

Since this paper was written, the authors have discovered that although Melin (1983)'s work did not provide the first-order expressions (52) of the SIF for a periodic array of slightly inclined cracks, it did contain formulae from which they may be deduced with some additional calculations. The confirmation thus brought to formulae (52) is both quite welcome in view of the complexity of the problem, and fully significant since Melin (1983)'s method, based on continuous distributions of dislocations, widely differed from that employed here.

\section{Acknowledgement}

The authors are indebted to Alain Karma of Northeastern University for having suggested to investigate the model problem considered in this paper, with applications to crack propagation in mode I+III in view. 


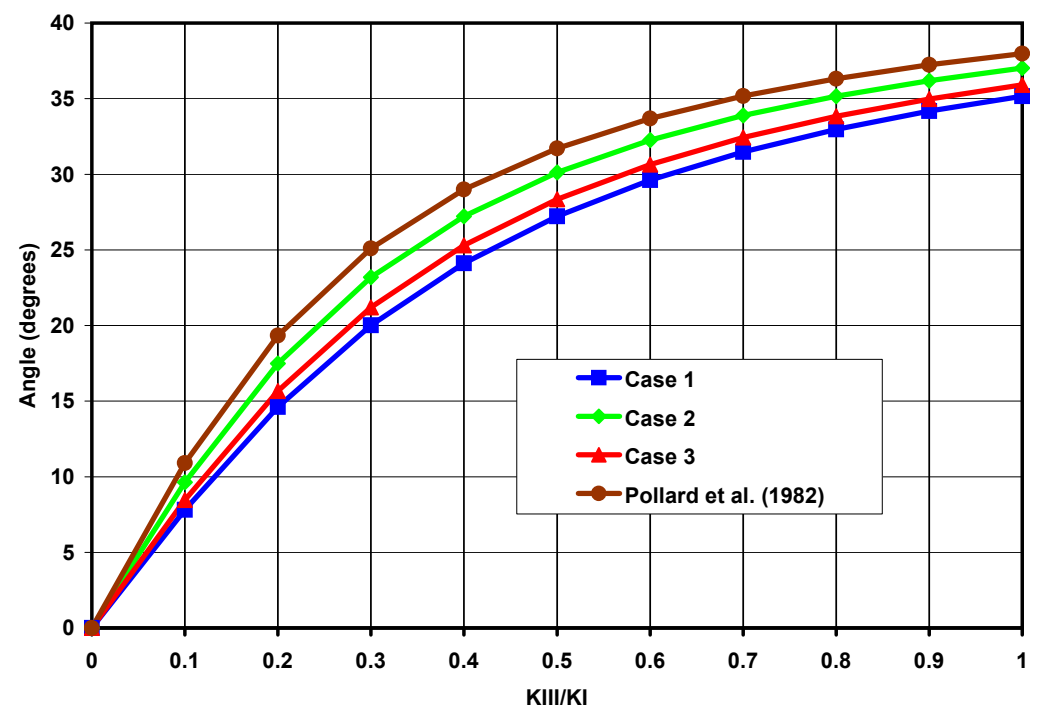

Fig. 12. The tilt angle as a function of the ratio $K_{I I I}^{3 D} / K_{I}^{3 D}$, for $\nu=0.25$ and $c / d=0.5$

\section{References}

Chen Y., Lin X. and Wang Z. (2009). Evaluation of the stress intensity factors and the T-stress in periodic crack problem. Int. J. Fracture, 156, 203-216.

Cooke M.L. and Pollard D.D. (1996). Fracture propagation paths under mixed mode loading within rectangular blocks of polymethyl methacrylate. J. Geophys. Res., 101, 3387-3400.

Destuynder P., Jaoua M. and Lescure S. (1983). Quelques remarques sur la mécanique de la rupture élastique. J. Méc. Théor. Appl., 2, 113-135 (in French).

Fleck N. (1991). Brittle fracture due to an array of microcracks. Proc. Roy. Soc. London $A, 432,55-76$.

Gao H. and Rice J.R. (1986). Shear stress intensity factors for planar crack with slightly curved front. ASME J. Appl. Mech., 53, 774-778.

Goldstein R.V. and Osipenko N.M. (2012). Successive development of the structure of a fracture near the front of a longitudinal shear crack. Doklady Physics, 57, 281-284.

Goldstein R.V. and Salganik R.L. (1974). Brittle fracture of solids with arbitrary cracks. Int. J. Fracture, 10, 507-523.

Griffith A.A. (1920). The phenomena of rupture and flow in solids. Phil. Trans. Roy. Soc. London, Series A, 221, 163-198.

Gradshteyn I.S. and Ryzhik I.M. (1980). Table of Integrals, Series, and Products, Academic Press, New York.

Hakim V. and Karma A. (2009). Laws of crack motion and phase-field models of fracture. J. Mech. Phys. Solids, 57, 342-368.

Hourlier F. and Pineau A. (1979). Fissuration par fatigue sous sollicitations polymodales (mode I ondulé + mode III permanent) d'un acier pour rotors 26NCDV14. Mémoires Scientifiques de la Revue de Métallurgie, 76, 175-185 (in French). 
Hubbard S. (1995). Determining the fatigue threshold and crack propagation properties of a steel under mixed mode I and III loading. M.Sc. Dissertation, Sheffield University, GB.

Hull D. (1993). Tilting cracks: the evolution of fracture surface topology in brittle solids. Int. J. Fracture, 62, 119-138.

Hull D. (1995). The effect of mixed mode I/III on crack evolution in brittle solids. Int. J. Fracture, 70, 59-79.

Karma A., Kessler D.A. and Levine H. (2001). Phase-field model of mode III dynamic fracture. Phys. Rev. Lett., 87, 045501 [4 pages].

Knauss W.G. (1970). An observation of crack propagation in antiplane shear. Int. J. Fracture, 6, 183-187.

Koiter W.T. (1959). An infinite row of parallel cracks in an infinite elastic sheet. IngenieurArchiv, 28, 168-172.

Lazarus V. (1997). Quelques problèmes tridimensionnels de mécanique de la rupture fragile. Ph.D. Thesis, Université Pierre et Marie Curie (Paris VI), France (in French).

Lazarus V., Buchholz F.G., Fulland M. and Wiebesiek J. (2008). Comparison of predictions by mode II or mode III criteria on crack front twisting in three or four point bending experiments. Int. J. Fracture, 153, 141-151.

Lazarus V., Leblond J.B. and Mouchrif S.E. (2001a). Crack front rotation and segmentation in mixed mode I+III or I+II+III - Part I: Calculation of stress intensity factors. J. Mech. Phys. Solids, 49, 1399-1420.

Lazarus V., Leblond J.B. and Mouchrif S.E. (2001b). Crack front rotation and segmentation in mixed mode I+III or I+II+III - Part II: Comparison with experiments, $J$. Mech. Phys. Solids, 49, 1421-1443.

Leblond J.B., Karma A. and Lazarus V. (2011). Theoretical analysis of crack front instability in mode I+III. J. Mech. Phys. Solids, 59, 1872-1887.

Lin B., Mear M.E. and Ravi-Chandar K. (2010). Criterion for initiation of cracks under mixed-mode I+III loading. Int. J. Fracture, 165, 175-188.

Melin S. (1983). Why do cracks avoid each other? Int. J. Fracture, 23, 37-45.

Movchan A.B., Gao H. and Willis J.R. (1998). On perturbations of plane cracks. Int. J. Solids Structures, 35, 3419-3453.

Muskhelishvili N.I. (1953). Some Basic Problems of the Mathematical Theory of Elasticity, Noordhoff, Groningen.

Palaniswamy K. and Knauss W.G. (1975). Crack extension in brittle solids. In: Mechanics Today, Vol. 4, Nemat-Nasser, Ed., Pergamon Press, pp. 87-148.

Pollard D.D. and Aydin A. (1988). Progress in understanding jointing over the past century. Geol. Soc. Amer. Bull., 100, 1181-1204.

Pollard D.D., Segall P. and Delaney P.T. (1982). Formation and interpretation of dilatant echelon cracks. Geol. Soc. Amer. Bull., 93, 1291-1303.

Pons A.J. and Karma A. (2010). Helical crack-front instability in mixed-mode fracture. Nature, 464, 85-89.

Sommer E. (1969). Formation of fracture "lances" in glass. Engng. Fracture Mech., 1, 539-546.

Suresh S. and Tschegg E.K. (1987). Combined mode I - mode III fracture of fatigueprecracked alumina. J. Amer. Ceramic Soc., 70, 726-733.

Tada H., Paris P. and Irwin G. (2000). The Stress Analysis of Cracks Handbook, 3rd edition, the American Society of Mechanical Engineers, New York. 
Wang G. and Feng X. (2001). The interaction of multiple rows of periodical cracks. Int. J. Fracture, 110, 73-100.

Westergaard H.M. (1939). Bearing pressures and cracks. ASME J. Appl. Mech. Series A, 61, 49-53.

Yates J.R. and Miller K.J. (1989). Mixed-mode (I+III) fatigue thresholds in a forging steel. Fatigue Fracture Engng. Materials Structures, 12, 259-270.

Yates J.R. and Mohammed R.A. (1994). Crack propagation under mixed mode (I+III) loading. Proc. 4th Int. Conf. on Biaxial/Multiaxial Fatigue, Saint-Germain-en-Laye, France, Vol. II, pp. 99-106. 


\section{A Appendix :}

The aim of this Appendix is to calculate the limit $L$ defined by equation (44).

The first step consists in looking for the asymptotic expressions of the function $q(t)$ near the tips $\pm c$. Using the expression $(36)_{2}$ of this function, one gets upon tedious but elementary calculations

$$
q(t)=\frac{ \pm k}{\sqrt{c \mp t}}+O(1) \quad \text { for } t \rightarrow \pm c
$$

where

$$
k \equiv i \sigma_{12}^{\infty} \sqrt{\frac{d}{\pi} \tan \left(\frac{\pi c}{2 d}\right)}\left[1+\frac{\pi c / d}{\sin (\pi c / d)}\right] .
$$

In a second step, the integral $\int_{-c}^{c}(\ldots)$ in the definition $(44)$ is rewritten as $\int_{-c}^{0}(\ldots)+\int_{0}^{c}(\ldots)$. In the integral $\int_{-c}^{0}(\ldots), \cot \left[\frac{\pi(c+r-t)}{2 d}\right]$ may be bounded by a constant, since $\frac{\pi(c+r)}{2 d} \leq \frac{\pi(c+r-t)}{2 d} \leq$ $\frac{\pi(2 c+r)}{2 d}$ so that for sufficiently small $r, m<\frac{\pi(c+r-t)}{2 d}<M$ where $0<m<M<\pi$. Hence the integral is bounded by a constant times $\sqrt{r} \int_{-c}^{0}|q(t)| d t$ which goes to zero with $r$ since $\int_{-c}^{0}|q(t)| d t<+\infty$ by equation (A.1). Thus equation (44) reduces to

$$
L=\lim _{r>0, r \rightarrow 0} \int_{0}^{c} \sqrt{r} \cot \left[\frac{\pi(c+r-t)}{2 d}\right] q(t) d t .
$$

In a third step, we note that if $0<x<M$ where $0<M<\pi$, then

$$
\cot x=\frac{1}{x}+h(x)
$$

where $h(x)$ is a bounded function. Now in the integral $\int_{0}^{c}(\ldots), \frac{\pi r}{2 d} \leq \frac{\pi(c+r-t)}{2 d} \leq \frac{\pi(c+r)}{2 d}$ so that $0<\frac{\pi(c+r-t)}{2 d}<\frac{3 \pi}{4}$ for sufficiently small $r$; thus one may use this decomposition of the cotangent. But the contribution arising from the function $h(x)$ in the integral goes to zero with $r$ since it is bounded by a constant times $\sqrt{r} \int_{0}^{c}|q(t)| d t$ with $\int_{0}^{c}|q(t)| d t<+\infty$. Hence equation (A.3) reduces to

$$
L=\lim _{r>0, r \rightarrow 0} \int_{0}^{c} \sqrt{r} \frac{2 d}{\pi(c+r-t)} q(t) d t
$$

In a fourth step, we note that by equation (A.1), if $0 \leq t \leq c$,

$$
q(t)=\frac{k}{\sqrt{c-t}}+q_{*}(t)
$$

where $q_{*}(t)$ is a bounded function. The contribution of this function in the integral of equation (A.4) is bounded by some constant times the integral $\int_{0}^{c} \frac{\sqrt{r}}{c+r-t} d t$ which is $O(\sqrt{r} \ln r)$ 
and therefore goes to zero with $r$. Hence equation (A.4) reduces to

$$
L=\lim _{r>0, r \rightarrow 0} \frac{2 d k}{\pi} \int_{0}^{c} \frac{\sqrt{r}}{(c+r-t) \sqrt{c-t}} d t .
$$

The fifth and final step consists in calculating the integral in equation (A.5) using the changes of variable $u \equiv c-t$, then $v \equiv u / r$ :

$$
\int_{0}^{c} \frac{\sqrt{r}}{(c+r-t) \sqrt{c-t}} d t=\int_{0}^{c} \frac{\sqrt{r}}{(u+r) \sqrt{u}} d u=\int_{0}^{c / r} \frac{d v}{(v+1) \sqrt{v}} .
$$

In the limit $r>0, r \rightarrow 0$, this integral goes to the limit

$$
\int_{0}^{+\infty} \frac{d v}{(v+1) \sqrt{v}}=\int_{0}^{+\infty} \frac{2 d w}{w^{2}+1}=\pi
$$

where the change of variable $w \equiv \sqrt{v}$ has been used. Therefore, by equation (A.5),

$$
L=2 d k,
$$

which yields equation (45) of the text upon use of the expression (A.2) of $k$. 